\title{
On the $K$-theory of division algebras over local fields
}

\author{
Lars Hesselholt · Michael Larsen · Ayelet Lindenstrauss
}

Andrei Suslin in memoriam

\section{Introduction}

Let $K$ be a complete discrete valuation field with finite residue field of characteristic $p$, and let $D$ be a central division algebra over $K$ of finite index $d$. Thirty years ago, Suslin and Yufryakov [35. Theorem 3] showed that for all prime numbers $\ell \neq p$ and integers $j \geqslant 1$, there exists an isomorphism of $\ell$-adic $K$-groups

$$
K_{j}\left(D, \mathbb{Z}_{\ell}\right) \stackrel{\operatorname{Nrd}_{D / K}}{\longrightarrow} K_{j}\left(K, \mathbb{Z}_{\ell}\right)
$$

such that $d \cdot \operatorname{Nrd}_{D / K}$ is equal to the norm homomorphism $N_{D / K}$. The purpose of this paper is to prove the following analogous result for the $p$-adic $K$-groups.

Theorem A. Let $D$ be a central division algebra of finite index $d$ over a complete discrete valuation field $K$ with finite residue field of odd characteristic $p$. For all integers $j \geqslant 1$, there exists a canonical isomorphism of p-adic $K$-groups

$$
K_{j}\left(D, \mathbb{Z}_{p}\right) \stackrel{\operatorname{Nrd}_{D / K}}{\longrightarrow} K_{j}\left(K, \mathbb{Z}_{p}\right)
$$

such that $d \cdot \operatorname{Nrd}_{D / K}$ is equal to the norm homomorphism $N_{D / K}$.

By contrast to the norm homomorphism $N_{D / K}$, we do not know that the reduced norm isomorphism $\operatorname{Nrd}_{D / K}$ is induced by a map of $K$-theory spectra, and such a map may well not exist; compare Merkurjev [29, Proposition 4].

Lars Hesselholt

Nagoya University, Nagoya, Japan and University of Copenhagen, Copenhagen, Denmark

E-mail: larsh@math.nagoya-u.ac.jp

Michael Larsen

Indiana University, Bloomington, Indiana

E-mail: mjlarsen@indiana.edu

Ayelet Lindenstrauss

Indiana University, Bloomington, Indiana

E-mail: alindens@indiana.edu 
The tool that makes it possible to now prove Theorem $\mathrm{A}$ is the topological cyclic homology introduced by Bökstedt, Hsiang, and Madsen [3]. The recent work by Nikolaus and Scholze [31] has greatly clarified the nature of this theory, and we will use their setup, which we briefly explain. Let $\mathrm{Sp}_{p}^{B T}$ be the infinity-category of $p$-complete spectra with an action by the circle $\mathbb{T}$. Given $X \in \mathrm{Sp}_{p}^{B \mathbb{T}}$, we write

$$
\mathrm{TC}^{-}(X) \stackrel{\text { can }}{\longrightarrow} \mathrm{TP}(X)
$$

for the canonical map from the homotopy fixed points spectrum to the Tate spectrum of $X$. We refer to these spectra as the negative topological cyclic homology and the periodic topological cyclic homology of $X$, respectively. A cyclotomic structure on $X$ is a map of spectra with $\mathbb{T}$-action

$$
X \stackrel{\varphi}{\longrightarrow} X^{t C_{p}}
$$

called the Frobenius map. The target of this map is the Tate spectrum of $X$ with respect to the subgroup $C_{p} \subset \mathbb{T}$ of order $p$. It has a residual $\mathbb{T} / C_{p}$-action, which we consider a $\mathbb{T}$-action via the $p$ th root isomorphism $\rho: \mathbb{T} \rightarrow \mathbb{T} / C_{p}$. The Frobenius induces a map of homotopy $\mathbb{T}$-fixed points,

$$
\mathrm{TC}^{-}(X)=X^{h \mathbb{T}} \stackrel{\varphi^{h \mathbb{T}}}{\longrightarrow}\left(X^{t C_{p}}\right)^{h\left(\mathbb{T} / C_{p}\right)} \simeq X^{t \mathbb{T}}=\operatorname{TP}(X),
$$

the target of which is canonically identified with the $\mathbb{T}$-Tate spectrum by the Tate orbit lemma of Nikolaus and Scholze [31, Lemma I.2.1]. We will abuse notation and write $\varphi: \mathrm{TC}^{-}(X) \rightarrow \mathrm{TP}(X)$ for the resulting map, which we again call the Frobenius map. Now, the topological cyclic homology of $X$ is the homotopy equalizer

$$
\mathrm{TC}(X) \stackrel{i}{\longrightarrow} \mathrm{TC}^{-}(X) \stackrel{\varphi}{\stackrel{\mathrm{can}}{\longrightarrow}} \mathrm{TP}(X)
$$

of the Frobenius map and the canonical map. Nikolaus and Scholze also show that, in a natural way, the $p$-complete cyclotomic spectra can be organized into a symmetric monoidal stable infinity-category $\mathrm{CycSp}_{p}$, whose tensor unit is the $p$-complete sphere spectrum $\mathbb{S}_{p}$ with trivial $\mathbb{T}$-action and with the composite map

$$
\mathbb{S}_{p} \stackrel{\tilde{\varphi}}{\longrightarrow} \mathbb{S}_{p}^{h C_{p}} \stackrel{\text { can }}{\longrightarrow} \mathbb{S}_{p}^{t C_{p}}
$$

as its cyclotomic structure map. Here, the left-hand map is the canonical map from a spectrum with trivial action to its fixed points. Finally, the left-hand map in the homotopy equalizer above is canonically identified with the "forgetful" map

$$
\operatorname{map}_{\mathrm{CycSp}_{p}}\left(\mathbb{S}_{p}, X\right) \stackrel{i}{\longrightarrow} \operatorname{map}_{\mathrm{Sp}_{p}^{B \mathbb{T}}}\left(\mathbb{S}_{p}, X\right)
$$

between mapping spectra in the stable infinity-categories of $p$-complete cyclotomic spectra and $p$-complete spectra with $\mathbb{T}$-action, respectively.

We now let $K$ be the quotient field of a complete discrete valuation ring $S$ with finite residue field $k_{S}$ of characteristic $p$, let $D$ be a central division algebra over $K$, and let $d=\operatorname{dim}_{K}(D)^{1 / 2}$ be the index of $D$ over $K$. The valuation on $K$ extends uniquely to 
a valuation on $D$, and the subring $A \subset D$ of elements of non-negative valuation is the unique maximal $S$-order. In [20], the first author and Madsen produced a $p$-complete cyclotomic spectrum $\operatorname{THH}\left(S \mid K, \mathbb{Z}_{p}\right)$ and a trace map

$$
K\left(K, \mathbb{Z}_{p}\right) \stackrel{\operatorname{tr}}{\longrightarrow} \mathrm{TC}\left(S \mid K, \mathbb{Z}_{p}\right)
$$

to its topological cyclic homology spectrum, which we abbreviate as indicated, from the $p$-completion of the algebraic $K$-theory spectrum of the field $K$. This construction also defines a $p$-complete cyclotomic spectrum $\operatorname{THH}\left(A \mid D, \mathbb{Z}_{p}\right)$ and a trace map

$$
K\left(D, \mathbb{Z}_{p}\right) \stackrel{\operatorname{tr}}{\longrightarrow} \mathrm{TC}\left(A \mid D, \mathbb{Z}_{p}\right)
$$

to its topological cyclic homology spectrum from the $p$-completion of the algebraic $K$-theory spectrum of the division ring $D$. Moreover, by [19, Theorem D], both maps induce isomorphisms of homotopy groups in degrees $j \geqslant 1$. Hence, Theorem $\mathrm{A}$ is a consequence of the following more precise Theorem B In order to state it, we first remark that $\operatorname{THH}\left(S \mid K, \mathbb{Z}_{p}\right)$ has a canonical structure of an $\mathbb{E}_{\infty}$-algebra in $\mathrm{CycSp}_{p}$, that $\operatorname{THH}\left(A \mid D, \mathbb{Z}_{p}\right)$ has a canonical structure of a $\operatorname{THH}\left(S \mid K, \mathbb{Z}_{p}\right)$-module, and that the trace map $\operatorname{Tr}_{A / S}$ is a map of $\operatorname{THH}\left(S \mid K, \mathbb{Z}_{p}\right)$-modules

$$
\operatorname{THH}\left(A \mid D, \mathbb{Z}_{p}\right) \stackrel{\operatorname{Tr}_{A / S}}{\longrightarrow} \mathrm{THH}\left(S \mid K, \mathbb{Z}_{p}\right)
$$

in $\mathrm{CycSp}_{p}$. The following is our main result.

Theorem B. Let $K$ be the quotient field of a complete discrete valuation ring $S$ with finite residue field of odd characteristic $p$, let $D$ be a central division algebra over $K$, and let $A \subset D$ be the maximal $S$-order.

(1) There exists an equivalence of $\operatorname{THH}\left(S \mid K, \mathbb{Z}_{p}\right)$-modules in cyclotomic spectra,

$$
\operatorname{THH}\left(A \mid D, \mathbb{Z}_{p}\right) \stackrel{\operatorname{Trd}_{A / S}}{\longrightarrow} \operatorname{THH}\left(S \mid K, \mathbb{Z}_{p}\right) .
$$

(2) As maps of $\mathrm{THH}\left(S \mid K, \mathbb{Z}_{p}\right)$-modules in spectra with $\mathbb{T}$-action,

$$
d \cdot \operatorname{Trd}_{A / S} \simeq \operatorname{Tr}_{A / S} .
$$

(3) If $p$ divides $d$, then, as maps of $\operatorname{THH}\left(S \mid K, \mathbb{Z}_{p}\right)$-modules in cyclotomic spectra,

$$
d \cdot \operatorname{Trd}_{A / S} \not 千 \operatorname{Tr}_{A / S} .
$$

The first part of the theorem shows, in particular, that $\mathrm{TC}_{*}\left(A \mid D, \mathbb{Z}_{p}\right)$ is free on a generator of degree 0 as a graded $\mathrm{TC}_{*}\left(S \mid K, \mathbb{Z}_{p}\right)$-module, and this implies the first statement in Theorem A This generator is not in the image of the cyclotomic trace, and $K_{*}\left(D, \mathbb{Z}_{p}\right)$ is neither free nor finitely generated as a graded $K_{*}\left(K, \mathbb{Z}_{p}\right)$-module.

To produce the desired equivalence of $\operatorname{THH}\left(S \mid K, \mathbb{Z}_{p}\right)$-modules in $\mathrm{CycSp}_{p}$, we instead produce its inverse equivalence

$$
\operatorname{THH}\left(S \mid K, \mathbb{Z}_{p}\right) \stackrel{\operatorname{Ird}_{A / S}}{\longrightarrow} \operatorname{THH}\left(A \mid D, \mathbb{Z}_{p}\right) .
$$


The space of such maps has group of components $\mathrm{TC}_{0}\left(A \mid D, \mathbb{Z}_{p}\right)$, and, similarly, the corresponding space of maps in $\mathrm{Sp}_{p}^{B \mathbb{T}}$ has group of components $\operatorname{TC}_{0}^{-}\left(A \mid D, \mathbb{Z}_{p}\right)$. To understand $\mathrm{TC}_{*}^{-}\left(A \mid D, \mathbb{Z}_{p}\right)$, we choose a maximal unramified subfield $K \subset L \subset D$ and let $S \subset T \subset A$ be the subring of elements of non-negative valuation. The extension $L / K$ is of degree $d$ and the Galois group $G$ of $L / K$ is canonically isomorphic to that of the extension $k_{T} / k_{S}$ of residue fields. In general, for $R$ a unital associative ring, we write $\mathscr{P}_{R}$ for the exact category of finitely generated projective left $R$-modules. In the case at hand, the ring homomorphisms

$$
A \otimes_{S} T \stackrel{\pi}{\longleftarrow} T \otimes_{S} T \stackrel{\delta}{\longrightarrow} T
$$

given by the canonical inclusion and the multiplication are finite locally free, and hence, we have the functors $\pi_{*}: \mathscr{P}_{A \otimes_{S} T} \rightarrow \mathscr{P}_{T \otimes_{S} T}$ and $\delta_{*}: \mathscr{P}_{T} \rightarrow \mathscr{P}_{T \otimes_{S} T}$ given by restriction-of-scalars along $\pi$ and $\delta$, respectively. Let $\pi^{!}$be the right adjoint of $\pi_{*}$ given by coextension-of-scalars along $\pi$, and let $\delta^{*}$ be the left adjoint of $\delta_{*}$ given by extension-of-scalars along $\delta$. We consider the diagram

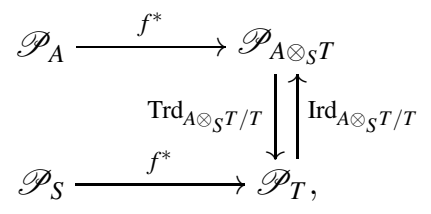

where the right-hand adjunction is the composite adjunction $\left(\delta^{*} \circ \pi_{*}, \pi^{!} \circ \delta_{*}\right)$, and where the horizontal functors are extension-of-scalars along the canonical inclusion $f: S \rightarrow T$. Said adjunction is not an adjoint equivalence of categories, but it becomes one after extension-of-scalars along the canonical inclusion $h: S \rightarrow K$, exhibiting the well-known Morita equivalence of $D \otimes_{K} L$ and $L$. The $\operatorname{ring} A \otimes_{S} T$ is not a maximal $T$-order in $D \otimes_{K} L$, so the following result, which we prove in Section 1 came as a rather fortunate surprise.

Theorem C. The ring $A \otimes_{S} T$ is left regular.

Here, we follow Bass [2, p. 122] and call a ring $R$ left regular if every finitely generated left $R$-module admits a finite resolution by finitely generated projective left $R$-modules. Using Theorem $\mathrm{C}$, we show that, in the diagram

$$
\begin{gathered}
\mathrm{TC}_{*}^{-}\left(A \mid D, \mathbb{Z}_{p}\right) \stackrel{f^{*}}{\longrightarrow} H^{0}\left(G, \mathrm{TC}_{*}^{-}\left(A \otimes_{S} T \mid D \otimes_{K} L, \mathbb{Z}_{p}\right)\right) \\
\operatorname{Trd}_{A \otimes_{S} T / T} \downarrow \uparrow \operatorname{Ird}_{A \otimes_{S} T / T} \\
\mathrm{TC}_{*}^{-}\left(S \mid K, \mathbb{Z}_{p}\right) \stackrel{f^{*}}{\longrightarrow} H^{0}\left(G, \mathrm{TC}_{*}^{-}\left(T \mid L, \mathbb{Z}_{p}\right)\right),
\end{gathered}
$$

the horizontal morphisms are isomorphisms, and the Morita equivalence mentioned above implies that also the vertical morphisms are isomorphisms. All morphisms in the diagram are graded $\mathrm{TC}_{*}^{-}\left(S \mid K, \mathbb{Z}_{p}\right)$-module homomorphisms, so we conclude that the graded $\mathrm{TC}_{*}^{-}\left(S \mid K, \mathbb{Z}_{p}\right)$-module $\mathrm{TC}_{*}^{-}\left(A \mid D, \mathbb{Z}_{p}\right)$ is free on a single generator of degree zero. We let $y \in \mathrm{TC}_{0}^{-}\left(A \mid D, \mathbb{Z}_{p}\right)$ be the unique generator with the property 
that $f^{*}(y)=\operatorname{Ird}_{A \otimes_{S} T / T}\left(f^{*}(1)\right)$. It satisfies that $\varphi(y)=\operatorname{can}(y)$, and accordingly, there exists $\tilde{y} \in \mathrm{TC}_{0}\left(A \mid D, \mathbb{Z}_{p}\right)$ with $i(\tilde{y})=y$. This implies part (1) of Theorem $\mathrm{B}$, and part (2) follows from the fact that $\operatorname{Tr}_{A / S}(y)=d \cdot 1$. This equation also characterizes the generator $y$, since the common groups $\mathrm{TC}_{0}^{-}\left(A \mid D, \mathbb{Z}_{p}\right)$ and $\mathrm{TC}_{0}^{-}\left(S \mid K, \mathbb{Z}_{p}\right)$ are free $\mathbb{Z}_{p}$-modules of rank one. By contrast, the common groups $\operatorname{TC}_{0}\left(A \mid D, \mathbb{Z}_{p}\right)$ and $\mathrm{TC}_{0}\left(S \mid K, \mathbb{Z}_{p}\right)$ are free $\mathbb{Z}_{p}$-modules of rank two, and we show that if $p$ divides $d$, then it is not possible to choose the generator $\tilde{y}$ such that $\operatorname{Tr}_{A / S}(\tilde{y})$ is a divisible by $d$, which implies part (3) of Theorem B.

The uniqueness of the generator $y \in \mathrm{TC}_{0}^{-}\left(A \mid D, \mathbb{Z}_{p}\right)$ with $\operatorname{Tr}_{A / S}(y)=d \cdot 1$ implies that the reduced trace isomorphisms on negative topological cyclic homology groups and on periodic cyclic homology groups are canonical and satisfy

$$
d \cdot \operatorname{Trd}_{A / S}=\operatorname{Tr}_{A / S}
$$

That the corresponding statements hold on topological cyclic homology groups is not immediately clear and is false in degree zero, if $p$ divides $d$. However, for $j \geqslant 1$ and odd, we show that there are exact sequences

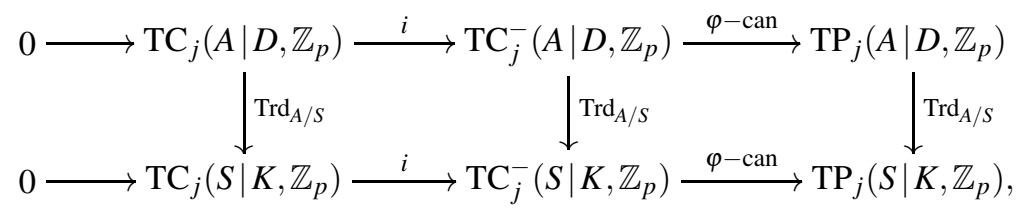

which show that also the left-hand map $\operatorname{Trd}_{A / S}$ is canonical and that its $d$ th multiple is equal to $\operatorname{Tr}_{A / S}$. Similarly, for $j \geqslant 2$ and even, there are exact sequences

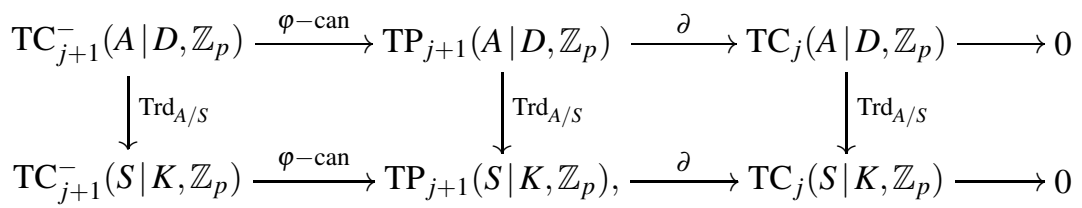

which show that the right-hand map $\operatorname{Trd}_{A / S}$ is canonical and that its $d$ th multiple is equal to $\operatorname{Tr}_{A / S}$. This proves that latter statement in Theorem $\mathrm{A}$ that the reduced norm isomorphism is canonical and satisfies $d \cdot \mathrm{Nrd}_{D / K}=N_{D / K}$.

Our proofs of Theorem $\mathrm{B}$ (2)-(3) use [20, Theorem 5.5.1], which is the reason that we assume $p$ to be odd. The remaining results hold also for $p=2$, as do all our results, if $S$ is of equal characteristic. We expect our results to hold also for $p=2$.

Acknowledgements We gratefully acknowledge the generous assistance that we have received from a DNRF Niels Bohr Professorship, Simons Foundation Grant 359565, and NSF Grants DMS-1702152 and DMS-1552766. The first author also thanks Indiana University and the Hausdorff Research Institute for Mathematics in Bonn for their hospitality and support, and the second and third author thank the University of Copenhagen for its hospitality and support. The first author further thanks Thomas Geisser for helpful discussions. We are much indebted to Jacob Lurie for pointing out a mistake in an earlier version of the work presented here, and finally, we thank an anonymous referee for many very helpful remarks. 


\section{Categories of modules}

In this section, we examine the structure of the category of left modules over the ring $A \otimes_{S} T$ and prove Theorem $\mathrm{C}$ of the introduction.

If $R$ is a unital associative ring $R$, then we write $\operatorname{Mod}_{R}$ for the category of left $R$-modules, and if $f: R \rightarrow S$ is a ring homomorphism, then we define the restriction along $f$ to be the functor $f_{*}: \operatorname{Mod}_{S} \rightarrow \operatorname{Mod}_{R}$ that to an $S$-module $M$ assigns the $R$ module $f_{*}(M)$ with the same underlying additive group as that of $M$ and with left scalar multiplication by $a \in R$ given by left scalar multiplication by $f(a) \in S$ and that to an $S$-linear map $h: M \rightarrow M^{\prime}$ assigns the same map $h: f_{*}(M) \rightarrow f_{*}\left(M^{\prime}\right)$. We note that the restriction of scalars along the identity homomorphism $\mathrm{id}_{R}$ and the identity functor of $\operatorname{Mod}_{R}$ are canonically naturally isomorphic, as are $(g \circ f)_{*}$ and $f_{*} \circ g_{*}$ for composable ring homomorphisms $f: R \rightarrow S$ and $g: S \rightarrow T$.

The functor $f_{*}$ admits both a left adjoint functor and a right adjoint functor. We say that a choice of an adjunction $\left(f^{*}, f_{*}, \varepsilon, \eta\right)$ from $\operatorname{Mod}_{R}$ to $\operatorname{Mod}_{S}$ is an extension of scalars along $f$; and we say that a choice of adjunction $\left(f_{*}, f^{!}, \varepsilon, \eta\right)$ from $\operatorname{Mod}_{R}$ to $\operatorname{Mod}_{S}$ is a coextension of scalars along $f$. If $f: R \rightarrow S$ and $g: S \rightarrow T$ are composable ring homomorphisms, then the functors $g^{*} \circ f^{*}$ and $(g \circ f)^{*}$ and the functors $g^{!} \circ f^{!}$ and $(g \circ f)^{!}$are canonically naturally isomorphic, and the extension and coextension along the identity homomorphism $\mathrm{id}_{R}$ both are canonically naturally isomorphic to the identity functor; compare [27, Theorem IV.7.2].

We write $\mathscr{M}_{R}$ and $\mathscr{P}_{R}$ for the full subcategories of $\operatorname{Mod}_{R}$ whose objects are the finitely generated left $R$-modules and the finitely generated projective left $R$-modules, respectively. Let $f: R \rightarrow S$ be a ring homomorphism. The extension of scalars along $f$ restricts to functors $f^{*}: \mathscr{M}_{R} \rightarrow \mathscr{M}_{S}$ and $f^{*}: \mathscr{P}_{R} \rightarrow \mathscr{P}_{S}$, the former of which is an exact functor if and only if $f$ is flat; the restriction of scalars along $f$ restricts to a functor $f_{*}: \mathscr{M}_{S} \rightarrow \mathscr{M}_{R}$, if $f$ is finite, and to a functor $f_{*}: \mathscr{P}_{S} \rightarrow \mathscr{P}_{R}$, if $f$ is finite and if $S$ considered as a left $R$-module via $f$ is projective, both of which are exact; and the coextension of scalars along $f$ restricts to exact functors $f^{!}: \mathscr{M}_{R} \rightarrow \mathscr{M}_{S}$, if $S$ is a finitely generated projective $R$-module, and $f^{!}: \mathscr{P}_{R} \rightarrow \mathscr{P}_{S}$, if, in addition, the coextension of $R, \operatorname{Hom}_{R}(S, R)$, is a finitely generated projective $S$-module. In particular, if $S$ is a finitely generated projective $R$-module and every object $M$ of $\mathscr{M}_{S}$ such that $f_{*} M$ is an object of $\mathscr{P}_{R}$ must in fact lie in $\mathscr{P}_{S}$, then $f^{!}: \mathscr{P}_{R} \rightarrow \mathscr{P}_{S}$ exists and is exact.

We again let $S$ be a complete discrete valuation ring with finite residue field $k_{S}$ of characteristic $p$ and with quotient field $K$, and let $D$ be a finite dimensional central division algebra over $K$. We recall the structure of $D$ following [34, Chapter 3]. The valuation $v_{K}$ on $K$ extends uniquely to a discrete valuation $v_{D}$ on $D$ given by

$$
v_{D}(x)=\frac{1}{\operatorname{dim}_{K}(D)} v_{K}\left(N_{D / K}(x)\right),
$$

where $x \in D^{*}$ and $N_{D / K}: D^{*} \rightarrow K^{*}$ is the norm. The algebra $D$ is complete with respect to $v_{D}$, and the subring $A \subset D$ of elements of non-negative valuation is both the integral closure of $S$ in $D$ and the unique maximal $S$-order in $D$. We choose a maximal subfield $K \subset L \subset D$ with the property that the extension $L / K$ is unramified and let $T \subset L$ be the integral closure of $S$. The equality $\operatorname{dim}_{K}(L)=\operatorname{dim}_{L}(D)$ holds, 
and the common dimension $d$ is called the index of $D$ over $K$. Hence, if $\pi_{D}$ is a generator of the unique maximal ideal $\mathfrak{m}_{D} \subset A$, then the tuple $\left(1, \pi_{D}, \ldots, \pi_{D}^{d-1}\right)$ is a basis of $D$ as a left $L$-vector space. Now, by [34, Theorem 14.5], we may choose the generator $\pi_{D}$ such that $\pi_{D}^{d}$ is contained in $S$ (and hence is a generator $\pi_{K}$ of the maximal ideal $\mathfrak{m}_{K} \subset S$ ) and such that the inner automorphism $x \mapsto \pi_{D} x \pi_{D}^{-1}$ of $D$ restricts to an automorphism $\sigma$ of $L / K$ which generates $\operatorname{Gal}(L / K)$. The map

$$
\operatorname{Gal}(L / K) \stackrel{\varepsilon}{\longrightarrow} \operatorname{Gal}\left(k_{T} / k_{S}\right)
$$

defined by $\varepsilon(g)\left(y+\mathfrak{m}_{T}\right)=g(y)+\mathfrak{m}_{T}$ is an isomorphism, since $L / K$ is unramified. It maps the generator $\sigma$ of the domain to a generator of the target, which we may therefore write as the $r$ th power of the Frobenius automorphism, for a unique integer $0<r<d$ relatively prime to $d$. The class of $r / d$ in $\mathbb{Q} / \mathbb{Z}$ is called the Hasse invariant of $D$. It determines the central division $K$-algebra $D$, up to non-canonical isomorphism. Moreover, every element of $\mathbb{Q} / \mathbb{Z}$ occurs as the index of some central division $K$-algebra $D$.

Let $k$ be a commutative ring, let $R$ be a commutative $k$-algebra, and let $\varphi: R \rightarrow R$ be a $k$-algebra automorphism. The twisted polynomial algebra $R^{\varphi}\{x\}$ is the quotient of the coproduct $R *_{k} k[x]$, in the category of unital associative $k$-algebras, of $R$ and $k[x]$ by the two-sided ideal generated by the family of elements $\varphi(a) x-x a$ with $a \in R$. We let $i_{1}: R \rightarrow R^{\varphi}\{x\}$ and $i_{2}: k[x] \rightarrow R^{\varphi}\{x\}$ be the two $k$-algebra homomorphisms defined as the compositions of the respective canonical inclusions into the coproduct followed by the canonical projection.

Lemma 1.1. In the situation above, if $k^{\prime}$ is a commutative $k$-algebra and $R^{\prime}=R \otimes_{k} k^{\prime}$, then there is a unique isomorphism of $k^{\prime}$-algebras

$$
\left(R^{\prime}\right)^{\sigma \otimes \mathrm{id}}\{x\} \stackrel{u}{\longrightarrow} R^{\sigma}\{x\} \otimes_{k} k^{\prime}
$$

compatible with the maps $i_{1}$ and $i_{2}$ over $k$ and $k^{\prime}$, respectively.

Proof. The universal property of coproducts gives a canonical map of $k^{\prime}$-algebras

$$
R^{\prime} *_{k^{\prime}} k^{\prime}[x] \longrightarrow\left(R *_{k} k[x]\right) \otimes_{k} k^{\prime},
$$

the universal property of extension of scalars gives a canonical map of $k$-modules

$$
\left(R *_{k} k[x]\right) \otimes_{k} k^{\prime} \longrightarrow R^{\prime} *_{k^{\prime}} k^{\prime}[x],
$$

and the two maps are mutually inverse. Moreover, the kernels of the canonical projections to $\left(R^{\prime}\right)^{\sigma \otimes \text { id }}\{x\}$ and $R^{\sigma}\{x\} \otimes_{k} k^{\prime}$, respectively, are identified under these isomorphisms.

Lemma 1.2. With notation as above, let $v$ be the unique S-algebra homomorphism

$$
T^{\sigma}\{x\} \stackrel{v}{\longrightarrow} A
$$

such that $v \circ i_{1}: T \rightarrow A$ is the canonical inclusion and $v \circ i_{2}: S[x] \rightarrow A$ is the unique $S$-algebra homomorphism mapping $x$ to $\pi_{D}$. Then $v$ is surjective, and its kernel is the two-sided ideal generated by $x^{d}-\pi_{K}$. 
Proof. To prove surjectivity, recall that every element $a \in D$ can be written uniquely as an $L$-linear combination $a=y_{0}+\cdots+y_{d-1} \pi_{D}^{d-1}$. Now, since $v_{D}\left(L^{\times}\right)=d \mathbb{Z}$, the values $v_{D}\left(y_{i} \pi_{D}^{i}\right)$ are pairwise distinct. Therefore, we conclude from the ultrametric inequality that $a \in A$ if and only if $y_{0}, \ldots, y_{d-1} \in T$ as desired. Clearly, $x^{d}-\pi_{K}$ lies in the kernel of $v$, and by the linear independence of $\left(1, \pi_{D}, \ldots, \pi_{D}^{d-1}\right)$ over $L$, no polynomial of lower degree does so. Therefore, by the right division algorithm, every element in the kernel of $v$ is a left multiple of the (central) element $x^{d}-\pi_{K}$.

Corollary 1.3. The unique T-algebra homomorphism

$$
\left(T \otimes_{S} T\right)^{\sigma \otimes \mathrm{id}}\{x\} \stackrel{v^{\prime}}{\longrightarrow} A \otimes_{S} T
$$

such that $v^{\prime} \circ\left(i_{1} \otimes \mathrm{id}\right): T \otimes_{S} T \rightarrow A \otimes_{S} T$ is the canonical inclusion and such that $v^{\prime} \circ\left(i_{2} \otimes \mathrm{id}\right): T[x] \rightarrow A \otimes_{S} T$ the unique $T$-algebra homomorphism that maps $x$ to $\pi_{D} \otimes 1$ is surjective, and its kernel is the two sided ideal $\left(x^{d}-\pi_{K} \otimes 1\right)$.

Proof. The map $v^{\prime}$ factors as the composition

$$
\left(T \otimes_{S} T\right)^{\sigma \otimes \mathrm{id}}\{x\} \stackrel{u}{\longrightarrow} T^{\sigma}\{x\} \otimes_{S} T \stackrel{v \otimes \mathrm{id}}{\longrightarrow} A \otimes_{S} T
$$

of the isomorphism in Lemma1.2 and the extension of scalars along $f: S \rightarrow T$ of the isomorphism in Lemma 1.3

We define a category $\operatorname{Mod}_{T, G}$ as follows. An object is a triple $\left(N,\left(N_{g}\right)_{g \in G}, \varphi\right)$, where $N$ is a left $T$-module, $\left(N_{g}\right)_{g \in G}$ is a grading on $N$ of type $G=\mathrm{Gal}(L / K)$, and $\varphi: N \rightarrow N$ is a graded $T$-linear endomorphism of degree $\sigma^{-1} \in G$ such that $\varphi^{d}$ is equal to left multiplication by $\pi_{K} \in T$. A morphism $h:\left(N,\left(N_{g}\right), \varphi\right) \rightarrow\left(N^{\prime},\left(N_{g}^{\prime}\right), \varphi^{\prime}\right)$ is a graded $T$-linear map $h: N \rightarrow N^{\prime}$ of degree $1 \in G$ such that $h \circ \varphi=\varphi^{\prime} \circ h$. We write the composition law in $G$ multiplicatively and refer to [6, Chapter $2, \S 11]$ for the definitions of graded modules and graded homomorphisms. We recall that since $f: S \rightarrow T$ is faithfully étale with Galois group $G$, the ring homomorphism

$$
T \otimes_{S} T \stackrel{w}{\longrightarrow} \prod_{g \in G} T
$$

with $g$ th component $w_{g}\left(t_{1} \otimes t_{2}\right)=g\left(t_{1}\right) t_{2}$ is an isomorphism. We let $\left(e_{g}\right)_{g \in G}$ be the family of orthogonal idempotents in $T \otimes_{S} T$ such that $w_{g}\left(e_{g^{\prime}}\right)=\delta_{g, g^{\prime}}$ and let

$$
\operatorname{Mod}_{A \otimes_{S} T} \stackrel{F}{\longrightarrow} \operatorname{Mod}_{T, G}
$$

be the functor that to a left $A \otimes_{S} T$-module $N$ assigns the triple $\left(N,\left(N_{g}\right)_{g \in G}, \varphi\right)$, where, by abuse of notation, $N$ is the underlying left $T$-module of the left $A \otimes_{S} T$-module $N$, where $N_{g} \subset N$ is the $T$-submodule $e_{g} \cdot N \subset N$, and where $\varphi: N \rightarrow N$ is the $T$-linear map given by left multiplication by $\pi_{D} \otimes 1 \in A \otimes_{S} T$.

Proposition 1.4. The functor

$$
\operatorname{Mod}_{A \otimes_{S} T} \stackrel{F}{\longrightarrow} \operatorname{Mod}_{T, G}
$$

is an equivalence of categories. 
Proof. First, to prove that $F$ is well-defined, we must verify that the $T$-linear map $\varphi: N \rightarrow N$ defined by $\varphi(y)=\pi_{D} \otimes 1 \cdot y$ is indeed graded of degree $\sigma^{-1}$ and that $\varphi^{d}$ is given by multiplication by $\pi_{K}$. The latter holds, since

$$
\pi_{D}^{d} \otimes 1=\pi_{K} \otimes 1=1 \otimes \pi_{K}
$$

and to prove the former we apply Corollary 1.3 to conclude that

$$
\varphi\left(\left(t_{1} \otimes t_{2}\right) y\right)=\left(\sigma\left(t_{1}\right) \otimes t_{2}\right) \varphi(y)
$$

for all $y \in N$ and $t_{1} \otimes t_{2} \in T \otimes_{S} T$. Moreover, the commutative diagram

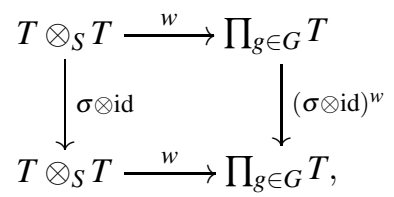

where the map $(\sigma \otimes \mathrm{id})^{w}$ is defined by $\operatorname{pr}_{g} \circ(\sigma \otimes \mathrm{id})^{w}=\mathrm{pr}_{g \circ \sigma}$, shows that

$$
(\sigma \otimes \mathrm{id})\left(e_{g}\right)=e_{g \circ \sigma^{-1}} .
$$

This shows that $\varphi: N \rightarrow N$ is graded of degree $\sigma^{-1}$ as desired.

We define a quasi-inverse functor

$$
\operatorname{Mod}_{T, G} \stackrel{H}{\longrightarrow} \operatorname{Mod}_{A \otimes S} T
$$

as follows. Given an object $\left(N,\left(N_{g}\right)_{g \in G}, \varphi\right)$ of the domain category, we first use the grading $\left(N_{g}\right)_{g \in G}$ to define a left $T \otimes_{S} T$-module structure on the left $T$-module $N$ by letting $t_{1} \otimes t_{2} \in T \otimes_{S} T$ multiply by $w_{g}\left(t_{1} \otimes t_{2}\right)$ on $N_{g} \subset N$. Moreover, since the $T$-linear map $\varphi: N \rightarrow N$ is graded of degree $\sigma^{-1}$, the argument above shows that

$$
\varphi\left(\left(t_{1} \otimes t_{2}\right) y\right)=\left(\sigma\left(t_{1}\right) \otimes t_{2}\right) \varphi(y)
$$

for all $y \in N$ and $t_{1} \otimes t_{2} \in T \otimes_{S} T$. Since, in addition, $\varphi^{d}$ is given by multiplication by $\pi_{K}$, this defines a $\left(T \otimes_{S} T\right)^{\sigma \otimes \text { id }}\{x\} /\left(x^{d}-\pi_{K} \otimes 1\right)$-module structure on $N$, where the left multiplication by $x$ is given by the map $\varphi: N \rightarrow N$, and by Corollary 1.3 , this defines a left $A \otimes_{S} T$-module structure on $N$. This defines the functor $H$, and it is clear that $F \circ H$ and $H \circ F$ are equal to the respective identity functors.

Example 1.5. Right multiplication on $A \otimes_{S} T$ by the idempotents $\left(e_{h}\right)_{h \in G}$ defined in the proof of Proposition 1.4 gives rise to a direct sum decomposition

$$
A \otimes_{S} T=\bigoplus_{h \in G} A \otimes_{S} T \cdot e_{h}
$$

as left $A \otimes_{S} T$-modules. Hence, as a left $A \otimes_{S} T$-module, each of the $d$ summands is projective. We now evaluate $F\left(A \otimes_{S} T \cdot e_{h}\right)$. By Corollary 1.3

$$
\left(\pi_{D} \otimes 1\right) \cdot e_{h}=(\sigma \otimes 1)\left(e_{h}\right) \cdot\left(\pi_{D} \otimes 1\right)=e_{h \circ \sigma^{-1}} \cdot\left(\pi_{D} \otimes 1\right),
$$


so as a left $T$-submodule of $A \otimes_{S} T$,

$$
e_{g} \cdot A \otimes_{S} T \cdot e_{h}=T \cdot\left(\pi_{D}^{i} \otimes 1\right)
$$

where $0 \leqslant i<d$ is the unique integer such that $g=h \circ \sigma^{-i}$. Hence, the map

$$
e_{g} \cdot A \otimes_{S} T \cdot e_{h} \stackrel{\varphi}{\longrightarrow} e_{g \circ \sigma^{-1}} \cdot A \otimes_{S} T \cdot e_{h}
$$

is an isomorphism, except for $g=h \circ \sigma$, where it is injective with cokernel $k_{T} \cdot 1$.

Let $\mathscr{M}_{T, G}$ and $\mathscr{P}_{T, G}$ be the full subcategories of $\operatorname{Mod}_{T, G}$ whose objects are the triples $\left(N,\left(N_{g}\right), \varphi\right)$ such that the left $T$-module $N$ is finitely generated and finitely generated and projective, respectively.

Addendum 1.6. The equivalence $F: \operatorname{Mod}_{A \otimes_{S} T} \rightarrow \operatorname{Mod}_{T, G}$ restricts to equivalences $F: \mathscr{M}_{A \otimes_{S} T} \rightarrow \mathscr{M}_{T, G}$ and $F: \mathscr{P}_{A \otimes_{S} T} \rightarrow \mathscr{P}_{T, G}$.

Proof. The $T$-algebra $A \otimes_{S} T$ is finitely generated and projective as a $T$-module. So the former statement follows immediately and to prove the latter, we must show that full subcategory $\mathscr{P}_{T, G}$ of $\mathscr{M}_{T, G}$ is precisely that consisting of the projective objects. Every projective object in $\mathscr{M}_{T, G}$ is an object in $\mathscr{P}_{T, G}$, since the underlying $T$-module of a projective left $A \otimes_{S} T$-module is projective. To prove the converse, let $\left(P,\left(P_{g}\right), \varphi\right)$ be an object of $\mathscr{P}_{T, G}$. The finitely generated projective $T$-modules $P_{g}$ all have the same rank $r$. Indeed, the $T$-linear map $\varphi^{d}: P_{g} \rightarrow P_{g}$ becomes an isomorphism after extending scalars along $T \rightarrow L$, and hence, so does $\varphi: P_{g} \rightarrow P_{g \circ \sigma^{-1}}$. Here and below we use that $\sigma \in G$ is a generator. We call the common rank $r$ the size of $\left(P,\left(P_{g}\right), \varphi\right)$ and proceed to show by induction on $r$ that $\left(P,\left(P_{g}\right), \varphi\right)$ is a projective object in $\mathscr{M}_{T, G}$, the case $r=0$ being trivial.

First, if $r=1$, then the $T$-modules $P_{g} / \pi_{K} P_{g}$ all have length 1 . It follows that the maps $\varphi: P_{g} \rightarrow P_{g \circ \sigma^{-1}}$ all are isomorphisms, except for a single $g=h \circ \sigma$ for which it is injective with cokernel of length 1 . We conclude from Example 1.5 that $\left(P,\left(P_{g}\right), \varphi\right)$ is isomorphic to the object $F\left(A \otimes_{S} T \cdot e_{h}\right)$, hence projective.

To prove the induction step, we let $\left(P,\left(P_{g}\right), \varphi\right)$ have size $r>1$ and assume that all objects of smaller size are projective. We will construct a sequence in $\mathscr{P}_{T, G}$,

$$
0 \longrightarrow\left(P^{\prime},\left(P_{g}^{\prime}\right), \varphi^{\prime}\right) \stackrel{j}{\longrightarrow}\left(P,\left(P_{g}\right), \varphi\right) \stackrel{q}{\longrightarrow}\left(P^{\prime \prime},\left(P_{g}^{\prime \prime}\right), \varphi^{\prime \prime}\right) \longrightarrow 0,
$$

which is exact in the abelian category $\mathscr{M}_{T, G}$ and in which the left-hand term has size 1. Inductively, the left-hand term and the right-hand term, which has size $r-1$, both are projective, and hence, the sequence will show that also the middle term is projective. To construct the desired sequence, we choose any non-zero element $x_{1} \in P_{1}$ and set $x_{g}=\varphi^{i}\left(x_{1}\right) \in P_{g}$ if $g=\sigma^{-i}$ with $0 \leqslant i<d$. We then define $j_{g}: P_{g}^{\prime} \rightarrow P_{g}$ by means of the pullback square of $T$-modules

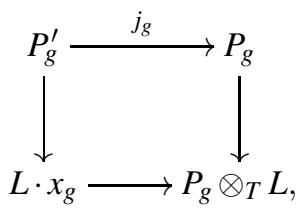


where all maps are the canonical inclusions, and define $q_{g}: P_{g} \rightarrow P_{g}^{\prime \prime}$ to be a cokernel of $j_{g}$. The $T$-modules $P_{g}^{\prime}$ and $P_{g}^{\prime \prime}$ are finitely generated of rank 1 and $r-1$, respectively, and both are torsion-free, and hence, projective. Here, to see that $P_{g}^{\prime \prime}$ is torsion-free, we use that if $x \in P_{g}$ and $p x \in P_{g}^{\prime}$, then $x \in P_{g}^{\prime}$. We define $j: P^{\prime} \rightarrow P$ and $q: P \rightarrow P^{\prime \prime}$ to be the respective sums indexed by $g \in G$ of $j_{g}: P_{g}^{\prime} \rightarrow P_{g}$ and $q_{g}: P_{g} \rightarrow P_{g}^{\prime \prime}$. The map $\varphi: P \rightarrow P$ induces maps $\varphi^{\prime}: P^{\prime} \rightarrow P^{\prime}$ and $\varphi^{\prime \prime}: P^{\prime \prime} \rightarrow P^{\prime \prime}$. Moreover, since $\varphi$ is graded $T$-linear of degree $\sigma^{-1}$ with $\varphi^{d}$ given by multiplication by $\pi_{K} \in T$, the same is true for the maps $\varphi^{\prime}$ and $\varphi^{\prime \prime}$. This completes the proof.

Corollary 1.7. Let $M$ be an $A \otimes_{S} T$-module. If the $T \otimes_{S} T$-module obtained from $M$ by restriction of scalars along $T \otimes_{S} T \rightarrow A \otimes_{S} T$ is finitely generated and projective, then $M$ is finitely generated and projective.

Proof. Since restriction of scalars along $T \rightarrow T \otimes_{S} T$ takes $\mathscr{P}_{T \otimes_{S} T}$ to $\mathscr{P}_{T}$, it suffices to prove that if the restriction of an $A \otimes_{S} T$-module $M$ along $T \rightarrow A \otimes_{S} T$ is finitely generated projective, then so is $M$. Applying $F$, this follows from the definition of $\mathscr{P}_{T, G}$ and from Addendum 1.6

Proof of Theorem $C$. We claim that the stronger statement that all submodules of a finitely generated projective left $A \otimes_{S} T$-module again are finitely generated projective holds. The analogous statement holds for the discrete valuation ring $T$. Hence, the claim follows from Addendum 1.6 and from $A \otimes_{S} T$ being noetherian.

We next identify the adjoint functors

$$
\mathscr{P}_{A \otimes S} T \stackrel{\operatorname{Tr}_{A \otimes S} T / T}{\stackrel{I_{A \otimes S^{T / T}}}{\rightleftarrows}} \mathscr{P}_{T}
$$

defined to be the restriction and coextension along $T \rightarrow A \otimes_{S} T$ under the equivalence of Addendum 1.6. Given $g \in G$, we define adjoint functors

$$
\mathscr{P}_{T, G} \underset{\text { ind }_{g}}{\stackrel{\operatorname{deg}_{g}}{\rightleftarrows}} \mathscr{P}_{T}
$$

by $\operatorname{deg}_{g}\left(P,\left(P_{h}\right)_{h \in G}, \varphi\right)=P_{g}$ and $\operatorname{ind}_{g}(Q)=\left(\bigoplus_{h \in G} Q,(Q)_{h \in G}, \psi\right)$, where $\psi$ takes the summand indexed by $h \in G$ to the one indexed by $h \circ \sigma^{-1} \in G$ by the map $\pi_{K} \cdot \operatorname{id}_{Q}$, if $h=g$, and by the identity map, otherwise. We define the adjunction isomorphism

$$
\operatorname{Hom}_{T}\left(\operatorname{deg}_{g}\left(P,\left(P_{h}\right)_{h \in G}, \varphi\right), Q\right) \stackrel{\alpha}{\longrightarrow} \operatorname{Hom}_{A \otimes_{S} T}\left(\left(P,\left(P_{h}\right)_{h \in G}, \varphi\right), \operatorname{ind}_{g}(Q)\right)
$$

by $\alpha(f)_{h}=f \circ \varphi^{i}: P_{h} \rightarrow Q$, where $g=h \circ \sigma^{-i}$ with $0 \leq i<d$.

Lemma 1.8. In the situation above, the following hold:

(i) The map induced by the canonical inclusions,

$$
\bigoplus_{g \in G}\left(\operatorname{deg}_{g} \circ F\right)(P) \longrightarrow \operatorname{Tr}_{A \otimes_{S} T / T}(P),
$$

is a natural isomorphism of left T-modules. 
(ii) Writing $g \in G$ as $g=\sigma^{-i}$ with $0 \leq i<d$, the $T$-linear map

$$
\left(\operatorname{deg}_{1} \circ F\right)(P) \stackrel{\left(\pi_{D} \otimes 1\right)^{i}}{\longrightarrow}\left(\operatorname{deg}_{g} \circ F\right)(P)
$$

is natural and becomes an isomorphism after extension of scalars along $T \rightarrow L$.

(iii) The multiplication $\delta: T \otimes_{S} T \rightarrow T$ induces a natural isomorphism

$$
\left(\operatorname{deg}_{1} \circ F\right)(P) \longrightarrow \operatorname{Trd}_{A \otimes_{S} T / T}(P) .
$$

Proof. This follows immediately from the definitions.

Remark 1.9. By adjunction, the statements (i)-(iii) in Lemma 1.8 imply that there is a natural isomorphism of left $A \otimes_{S} T$-modules

$$
I_{A \otimes_{S} T / T}(Q) \longrightarrow \prod_{g \in G}\left(H \circ \operatorname{ind}_{g}\right)(Q)
$$

that there is a natural $A \otimes_{S} T$-linear map

$$
\left(H \circ \operatorname{ind}_{g}\right)(Q) \longrightarrow\left(H \circ \operatorname{ind}_{1}\right)(Q)
$$

which becomes an isomorphism after extension of scalars along $A \otimes_{S} T \rightarrow D \otimes_{K} L$; and that there is a natural isomorphism of left $A \otimes_{S} T$-modules

$$
\operatorname{Ird}_{A \otimes_{S} T}(Q) \longrightarrow\left(H \circ \operatorname{ind}_{1}\right)(Q)
$$

Finally, we compare the $T$-order $A \otimes_{S} T$ in the semisimple $L$-algebra $D \otimes_{K} L$ to a maximal $T$-order. We recall from [34, Theorem 7.15] that, by viewing $D$ as a right $L$-vector space, left multiplication by $D$ on itself defines an $L$-algebra isomorphism

$$
D \otimes_{K} L \stackrel{l}{\longrightarrow} \operatorname{End}_{L}(D)
$$

It restricts to a $T$-algebra monomorphism from the $T$-order $A \otimes_{S} T$ of the domain to the $T$-order $\operatorname{End}_{T}(A)$ of the target, which by op. cit., Theorem 8.7, is a maximal $T$-order. We identify the $T$-algebra $\operatorname{End}_{T}(A)$ with the matrix $T$-algebra $M_{d}(T)$ by means of the $T$-algebra isomorphism $\operatorname{End}_{T}(A) \rightarrow M_{d}(T)$ that to an endomorphism of the right $T$-module $A$ associates its matrix with respect to the basis $\left(\pi_{D}^{s}\right)_{0 \leqslant s<d}$.

Proposition 1.10. With notation as above, there is a cartesian square of T-algebras

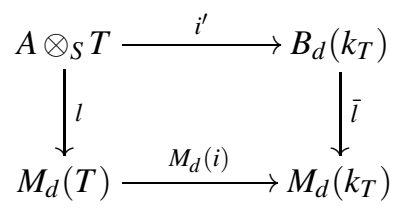

in which the right-hand vertical map is the canonical inclusion of the subalgebra of lower triangular matrices, and the map $i: T \rightarrow k_{T}$ is the canonical projection. 
Proof. The morphism $l$ maps the $T$-subalgebra $T \otimes_{S} T \subset A \otimes_{S} T$ isomorphically onto the $T$-subalgebra $H_{d}(T) \subset M_{d}(T)$ of diagonal matrices, and

$$
l\left(\pi_{D} \otimes 1\right)=\left(\begin{array}{cccccc}
0 & 0 & 0 & \cdots & 0 & \pi_{K} \\
1 & 0 & 0 & \cdots & 0 & 0 \\
0 & 1 & 0 & \cdots & 0 & 0 \\
\vdots & \vdots & \vdots & \ddots & \vdots & \vdots \\
0 & 0 & 0 & \cdots & 1 & 0
\end{array}\right) .
$$

Thus, the left $H_{d}(T)$-submodule of $M_{d}(T)$ spanned by $\left(l\left(\pi_{D}^{s} \otimes 1\right)\right)_{0 \leqslant s<d}$ is equal to the left $H_{d}(T)$-submodule of matrices that are lower triangular modulo $M_{d}\left(\mathfrak{m}_{T}\right)$, and, by Corollary 1.3 this left $H_{d}(T)$-submodule, in turn, is is equal to the image of the $T$-algebra homomorphism $l: A \otimes_{S} T \rightarrow M_{d}(T)$.

Remark 1.11. The top horizontal morphism $i^{\prime}$ in Proposition 1.10 maps the radical $\mathfrak{m}_{D} \otimes_{S} T \subset A \otimes_{S} T$ onto the nilpotent two-sided ideal $N_{d}\left(k_{T}\right) \subset B_{d}\left(k_{T}\right)$ of strictly lower triangular matrices. Moreover, the composition

$$
A \otimes_{S} T \stackrel{i^{\prime}}{\longrightarrow} B_{d}\left(k_{T}\right) \longrightarrow B_{d}\left(k_{T}\right) / N_{d}\left(k_{T}\right) \longrightarrow k_{T} \otimes_{k_{S}} k_{T}
$$

of $i^{\prime}$, the canonical projection, and the inverse of the isomorphism that maps $t_{1} \otimes t_{2}$ to the class of the diagonal matrix $\operatorname{diag}\left(t_{1} t_{2}, \sigma^{-1}\left(t_{1}\right) t_{2}, \ldots, \sigma^{-(d-1)}\left(t_{1}\right) t_{2}\right)$ is equal to the canonical projection $i: A \otimes_{S} T \rightarrow k_{T} \otimes_{k_{S}} k_{T}$.

\section{Localization sequences in $K$-theory and topological cyclic homology}

In this section, we recall the algebraic $K$-theory symmetric spectrum of a pointed exact category with weak equivalences following Waldhausen [37, Section 1] and prove a localization sequence needed in the proof of Theorem $B$

An exact category with weak equivalences is a triple $(\mathscr{C}, \mathscr{E}, \mathscr{W})$ of a category $\mathscr{C}$; a set $\mathscr{E}$ of exact sequences in $\mathscr{C}$ satisfying axioms (a) and (b) in [33, §2], axiom (c) being redundant [21, Appendix A]; and a subcategory $\mathscr{W}$ of weak equivalences in $\mathscr{C}$ satisfying axioms (Weq 1) and (Weq 2) in [37, Section 1.2]. An exact functor $F:(\mathscr{C}, \mathscr{E}, \mathscr{W}) \rightarrow\left(\mathscr{C}^{\prime}, \mathscr{E}^{\prime}, \mathscr{W}^{\prime}\right)$ between exact categories with weak equivalences is a functor $F: \mathscr{C} \rightarrow \mathscr{C}^{\prime}$ that maps $\mathscr{E}$ to $\mathscr{E}^{\prime}$ and $\mathscr{W}$ to $\mathscr{W}^{\prime}$; and an exact natural transformation between two such functors is a natural transformation $f: F \Rightarrow F^{\prime}$ such that for every object $c$ in $\mathscr{C}$, the morphism $f_{c}: F(c) \rightarrow F^{\prime}(c)$ belongs to $\mathscr{W}^{\prime}$. Now Waldhausen's $S$-construction is a functor

$$
\mathrm{ExCat} \stackrel{S}{\longrightarrow} \mathrm{ExCat}^{\mathrm{sp}}
$$

that to an exact category with weak equivalences assigns a simplicial exact category with weak equivalences. The construction, thus, may be iterated and gives, for every non-negative integer $r$, a functor

$$
\mathrm{ExCat} \stackrel{S^{r}}{\longrightarrow} \operatorname{ExCat}{ }^{\left(\Delta^{\mathrm{op}}\right)^{r}}
$$


that to an exact category with weak equivalences assigns an $r$-simplicial exact category with weak equivalences.

In the following, we will also write $w(\mathscr{C}, \mathscr{E}, \mathscr{W})$ instead of $\mathscr{W}$ for the subcategory of weak equivalences. We define

$$
\left(\mathscr{C}^{w}, \mathscr{E} \cap \mathscr{C}^{w}, \mathscr{W} \cap \mathscr{C}^{w}\right) \subset(\mathscr{C}, \mathscr{E}, \mathscr{W})
$$

be the full sub-exact category with weak equivalences consisting of those objects $c$ in $\mathscr{C}$ with the property that $0 \rightarrow c$ is in $\mathscr{W}$. Its subcategory of weak equivalences has a zero object, and therefore, is contractible. In particular, the subspace

$$
\left|N\left(w\left(\mathscr{C}^{w}, \mathscr{E} \cap \mathscr{C}^{w}, \mathscr{W} \cap \mathscr{C}^{w}\right)\right)\right| \subset|N(w(\mathscr{C}, \mathscr{E}, \mathscr{W}))|
$$

is contractible, and the pointed space given by the quotient

$$
K(\mathscr{C}, \mathscr{E}, \mathscr{W})_{r}=\left|N\left(w S^{r}(\mathscr{C}, \mathscr{E}, \mathscr{W})\right)\right| /\left|N\left(w S^{r}\left(\mathscr{C}^{w}, \mathscr{E} \cap \mathscr{C}^{w}, \mathscr{W} \cap \mathscr{C}^{w}\right)\right)\right|
$$

is by definition the $r$ th space in the symmetric spectrum $K(\mathscr{C}, \mathscr{E}, \mathscr{W})$. The left action by the symmetric group $\Sigma_{r}$ on this pointed space is induced from the permutation of the $r$-simplicial directions; and the spectrum structure maps

$$
K(\mathscr{C}, \mathscr{E}, \mathscr{W})_{r} \wedge S^{s} \stackrel{\sigma_{r, s}}{\longrightarrow} K(\mathscr{C}, \mathscr{E}, \mathscr{W})_{r+s}
$$

are induced by the inclusion of the 1-skeleta in the last $s$ simplicial directions. We refer to [13, Appendix] for proof that this is indeed a symmetric spectrum and for a discussion of multiplicative properties of the construction. We also recall that, as a consequence of the additivity theorem [37, Theorem 1.4.2, Proposition 1.5.3], the symmetric spectrum $K(\mathscr{C}, \mathscr{E}, \mathscr{W})$ is fibrant in the positive model structure on the category of symmetric spectra; see [28, Section 14].

An exact functor $F:(\mathscr{C}, \mathscr{E}, \mathscr{W}) \rightarrow\left(\mathscr{C}^{\prime}, \mathscr{E}^{\prime}, \mathscr{W}^{\prime}\right)$ induces a morphism

$$
K(\mathscr{C}, \mathscr{E}, \mathscr{W}) \stackrel{K(F)}{\longrightarrow} K\left(\mathscr{C}^{\prime}, \mathscr{E}^{\prime}, \mathscr{W}^{\prime}\right),
$$

of symmetric spectra and an exact natural transformations $f: F \Rightarrow F^{\prime}$ between two such functors gives rise to a homotopy $K(f)$ from $K(F)$ to $K\left(F^{\prime}\right)$. In this way, the $K$-theory construction is a strict 2-functor from the strict 2-category of exact categories with weak equivalences, exact functors, and exact natural transformations to the strict 2-category of symmetric spectra, morphisms of symmetric spectra, and homotopy classes of homotopies between morphisms of symmetric spectra. Like every 2 -functor, it takes adjunctions in the domain 2-category to adjunctions in the target 2-category, and the latter adjunctions automatically are adjoint equivalences, since the 2-morphisms in the target 2-category are invertible.

An abelian category $\mathscr{M}$ has a canonical structure of an exact category with weak equivalences, where the set of exact sequences $\mathscr{E}$ consists of the sequences

$$
M^{\prime} \stackrel{i}{\longrightarrow} M \stackrel{p}{\longrightarrow} M^{\prime \prime}
$$

in $\mathscr{M}$ such that $i$ is a kernel of $p$ and $p$ a cokernel of $i$, and where the subcategory of weak equivalences $\mathscr{W}$ is the subcategory of isomorphisms in $\mathscr{M}$. Moreover, an 
additive full subcategory $\mathscr{P}$ of $\mathscr{M}$, which is extension-closed in the sense that, for every sequence in $\mathscr{E}$ whose initial term $M^{\prime}$ and terminal term $M^{\prime \prime}$ are in $\mathscr{P}$, also the middle term $M$ is in $\mathscr{P}$, has an induced structure of exact category with weak equivalences, where the set of exact sequences $\mathscr{E} \cap \mathscr{P}$ consists of the sequences in $\mathscr{E}$ all of whose terms are in $\mathscr{P}$, and where the subcategory of weak equivalences $\mathscr{W} \cap \mathscr{P}$ is the full subcategory of $\mathscr{W}$ whose objects are in $\mathscr{P}$.

Now let $R$ be a left noetherian ring, and let $\mathscr{M}_{R}$ and $\mathscr{P}_{R}$ be the categories of finitely generated left $R$-modules and finitely generated and projective left $R$-modules, respectively. We assume that these categories are small, which may be accomplished by assuming the axiom of universe [1, Exposé] or by some ad hoc restriction on the modules allowed. The category $\mathscr{M}_{R}$ is abelian and the additive full subcategory $\mathscr{P}_{R}$ is extension-closed. We define $K^{\prime}(R)$ and $K(R)$ to be the $K$-theory symmetric spectra of the exact categories with weak equivalences associated to these as discussed above. We recall that the canonical inclusion functor induces a weak equivalence

$$
K(R) \longrightarrow K^{\prime}(R)
$$

provided that $R$ is left regular in the sense that every object in $\mathscr{M}_{R}$ admits a finite resolution by objects in $\mathscr{P}_{R}$.

Proposition 2.1. Let $S$ be a complete discrete valuation ring with residue field $k_{S}$ and quotient field $K$ and let $R$ be an $S$-algebra. Assuming that, as a ring, $R$ is left regular, there is a canonical natural cofibration sequence of symmetric spectra

$$
K^{\prime}\left(R \otimes_{S} k_{S}\right) \stackrel{i_{*}}{\longrightarrow} K(R) \stackrel{j^{*}}{\longrightarrow} K\left(R \otimes_{S} K\right) \stackrel{\partial}{\longrightarrow} \Sigma K^{\prime}\left(R \otimes_{S} k_{S}\right) .
$$

The terms in the sequence have canonical natural $K(S)$-module structures and the maps in the sequences respect these structures.

Proof. We first introduce some notation. Let $\mathscr{M}$ be an abelian category, let $\mathscr{P}$ be an extension-closed full additive subcategory of $\mathscr{M}$, and let $\mathscr{H}$ and $\mathscr{T}$ be two Serre subcategories of $\mathscr{M}$. Let $\mathscr{E} \cap \mathscr{P}$ be the set of exact sequences in the exact category structure on $\mathscr{P}$ defined above. We define $\mathrm{Ch}^{b}(\mathscr{P}, \mathscr{H}, \mathscr{T})$ to be the following exact category with weak equivalences: The underlying category has objects the bounded chain complexes in $\mathscr{P}$ whose associated homology objects, calculated in $\mathscr{M}$, are in the Serre subcategory $\mathscr{H}$, and has morphisms all chain maps; the exact sequences in this category are the sequences of complexes that degree-wise are in $\mathscr{E} \cap \mathscr{P}$; and the weak equivalences are the morphisms that, modulo the Serre subcategory $\mathscr{T}$, induce isomorphisms of homology objects. If $\mathscr{T}$ is the Serre subcategory of zero objects in $\mathscr{M}$, then we write $\mathrm{Ch}^{b}(\mathscr{P}, \mathscr{H})$ instead of $\mathrm{Ch}^{b}(\mathscr{P}, \mathscr{H}, \mathscr{T})$.

We now let $\mathscr{T}_{R}$ be the Serre subcategory of $\mathscr{M}_{R}$ whose objects are the finitely generated left $R$-modules annihilated by extension of scalars along $f: S \rightarrow K$ and consider the diagram of $K$-theory symmetric spectra

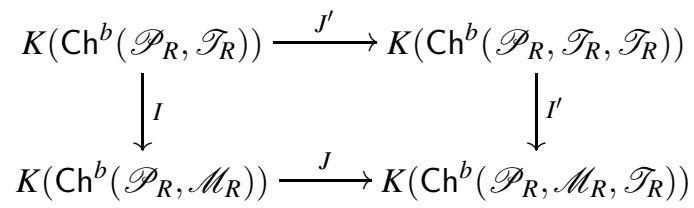


with all maps induced by the respective canonical inclusion functors. It follows from Waldhausen's fibration theorem [37, Theorem 1.6.4] that the diagram is homotopy cartesian. Moreover, by the 2 -functoriality of the $K$-theory construction, the unique natural transformation from the identity functor to a constant functor with value a zero object defines a homotopy from the identity map of the upper right-hand term to the constant map. Indeed, this natural transformation is exact. This homotopy, in turn, determines a homotopy from the composite map $J \circ I$ to the constant map, and the combined data determines a cofibration sequence in the homotopy category of symmetric spectra. We proceed to identify the terms and maps in this cofibration sequence with the ones in the cofibration sequence in the statement.

We use Waldhausen's approximation theorem [37, Theorem 1.6.7], but for our purposes, the formulation in [36, Theorem 1.9.8] is more convenient. The theorem states that the map of $K$-theory symmetric spectra induced by an exact functor $F$ is a weak equivalence, if a list of hypotheses are satisfied. In our situation, the only hypothesis that is not automatically satisfied is loc. cit. 1.9.7.1, which is the requirement that, for every object $B$ in the target of $F$, there exists an object $A$ in the domain of $F$ and a weak equivalence $f: F(A) \rightarrow B$ in the target of $F$.

Now, the left-hand vertical map $I$ fits in the diagram

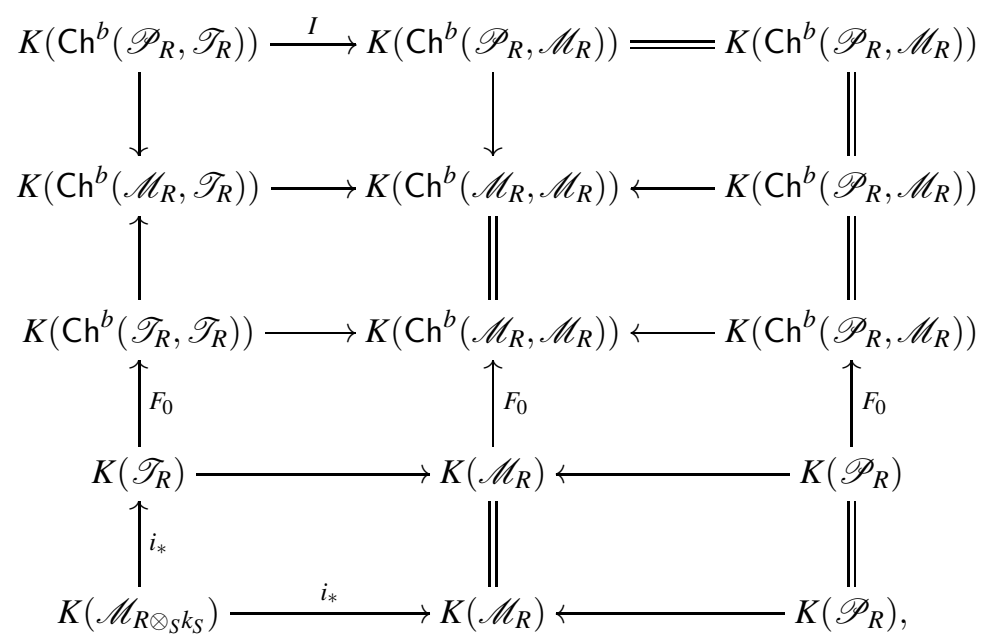

where the unmarked maps are induced by the respective canonical inclusion functors. The top vertical maps and the second and third right-hand horizontal maps are weak equivalences by op. cit. Theorem 1.9.8, and the vertical maps labelled $F_{0}$ are weak equivalences by op. cit. Theorem 1.11.7. The second left-hand vertical map also is a weak equivalence by op. cit. Theorem 1.9.8, the hypothesis 1.9.7.1 being satisfied by [20, Lemma 1.5.3]. Finally, the lower left-hand vertical map is a weak equivalence by Quillen's devissage theorem [33, Theorem 4]. 
Similarly, the lower horizontal map $J$ in the diagram above fits in the diagram

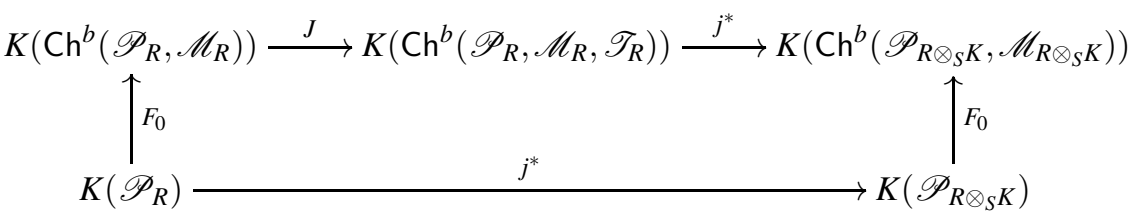

with the vertical maps induced by the exact functor that to a module $M$ assigns the complex $F_{0}(M)$ whose degree $n$ term is $M$, if $n=0$, and a zero object 0 , otherwise. The upper right-hand horizontal map is induced by the exact functor given by degreewise extension of scalars along $f: S \rightarrow K$, and it is a weak equivalence, since this functor satisfies the hypotheses op. cit. Theorem 1.9.8. The vertical maps also are weak equivalences by op. cit. Theorem 1.11.7.

We will prove an analogue of Proposition 2.1 for topological cyclic homology, and begin by recalling the definition following [12]. We denote by $\mathbb{T}$ the circle group of complex numbers of modulus 1 under multiplication.

For $(\mathscr{C}, \mathscr{E}, \mathscr{W})$ an exact category with weak equivalences, the Bökstedt-Dennis trace map is a natural morphism of symmetric spectra with left $\mathbb{T}$-action

$$
K(\mathscr{C}, \mathscr{E}, \mathscr{W}) \stackrel{\operatorname{tr}}{\longrightarrow} \mathrm{THH}(\mathscr{C}, \mathscr{E}, \mathscr{W})
$$

from the $K$-theory symmetric spectrum with trivial left $\mathbb{T}$-action to the topological Hochschild spectrum, the definition and properties of which we now briefly discuss. The topological Hochschild construction assigns to an additive category $\mathscr{C}$ the left $\mathbb{T}$-space $\operatorname{THH}(\mathscr{C})$ defined to be the realization of the cyclic space $\mathrm{THH}(\mathscr{C})[-]$ given in [12, Definition 1.3.6]. The left ' $\mathbb{T}$-action is a consequence of Connes' theory of cyclic objects [10], which also identifies the canonical inclusion of the subspace of points fixed by the left $\mathbb{T}$-action with a $\mathbb{T}$-equivariant map

$$
\mathrm{ob}(\mathscr{C}) \stackrel{\operatorname{tr}}{\longrightarrow} \mathrm{THH}(\mathscr{C})
$$

from the set of objects in $\mathscr{C}$ considered as a discrete space with trivial left $\mathbb{T}$-action. The topological Hochschild symmetric spectrum of an exact category with weak equivalences $(\mathscr{C}, \mathscr{E}, \mathscr{W})$ and the Bökstedt-Dennis trace map is defined by incorporating Waldhausen's $S$-construction as follows. If $I$ is a small category, then we define $(\mathscr{C}, \mathscr{E}, \mathscr{W})^{I}$ to be the exact category with the category $\mathscr{C}^{I}$ of I-diagrams in $\mathscr{C}$ as underlying category; with the sequences in $\mathscr{C}^{I}$ that, objectwise, are in $\mathscr{E}$ as the exact sequences; and with the morphisms in $\mathscr{C}^{I}$ that, objectwise, are in $\mathscr{W}$ as the weak equivalences. We also fix the fully faithful functor

$$
i: \Delta \rightarrow \text { Cat }
$$

that to a non-empty finite ordinal $[n]$ assigns the category $i([n])$ with object set $[n]$ and with a unique morphism from $s$ to $t$ if and only if $s \leqslant t$, and that to an order-preserving 
map $\theta:[m] \rightarrow[n]$ assigns the unique functor $i(\theta): i([m]) \rightarrow i([n])$ with the map $\theta$ as the underlying map of object sets. With these preparations in hand, we let

$$
N^{w}(\mathscr{C}, \mathscr{E}, \mathscr{W})[-] \subset(\mathscr{C}, \mathscr{E}, \mathscr{W})^{i([-])}
$$

be the sub-simplicial exact category with weak equivalences, whose underlying simplicial category is the full sub-simplicial category of $\mathscr{C}^{i([-])}$ with simplicial set of objects given by ob $\left(\mathscr{W}^{i([-])}\right)$. Again, the subspace

$$
\left|\operatorname{THH}\left(N^{w}\left(S^{r}\left(\mathscr{C}^{w}, \mathscr{E} \cap \mathscr{C}^{w}, \mathscr{W} \cap \mathscr{C}^{w}\right)\right)\right)\right| \subset\left|\operatorname{THH}\left(N^{w}\left(S^{r}(\mathscr{C}, \mathscr{E}, \mathscr{W})\right)\right)\right|
$$

is $\mathbb{T}$-equivariantly contractible, and the $r$ th space in the symmetric spectrum with left $\mathbb{T}$-action $\operatorname{THH}(\mathscr{C}, \mathscr{E}, \mathscr{W})$ is defined to be the quotient pointed left $\mathbb{T}$-space. Here, the topological Hochschild construction is applied degreewise to the $(r+1)$-simplicial additive categories in question. The structure maps

$$
\operatorname{THH}(\mathscr{C}, \mathscr{E}, \mathscr{W})_{r} \wedge S^{s} \stackrel{\sigma_{r, s}}{\longrightarrow} \operatorname{THH}(\mathscr{C}, \mathscr{E}, \mathscr{W})_{r+s}
$$

are defined analogously to those in the $K$-theory symmetric spectrum.

We next recall the classical definition of the Frobenius maps

$$
\operatorname{THH}(\mathscr{C}, \mathscr{E}, \mathscr{W}) \stackrel{\varphi_{p}}{\longrightarrow} \operatorname{THH}(\mathscr{C}, \mathscr{E}, \mathscr{W})^{t C_{p}}
$$

following [31, Section III.5] ] We define maps of symmetric spectra with left $\mathbb{T}$-action

$$
\operatorname{THH}(\mathscr{C}, \mathscr{E}, \mathscr{W}) \stackrel{r_{p}}{\longleftarrow} \rho_{p}^{*} \operatorname{THH}(\mathscr{C}, \mathscr{E}, \mathscr{W})^{\phi C_{p}} \stackrel{s_{p}}{\longrightarrow} \rho_{p}^{*} \operatorname{THH}(\mathscr{C}, \mathscr{E}, \mathscr{W})^{t C_{p}},
$$

where $C_{p} \subset \mathbb{T}$ is the subgroup of prime order $p$, and where $\mathbb{T}$ acts on the middle and right-hand terms via the $p$ th root isomorphism $\rho_{p}: \mathbb{T} \rightarrow \mathbb{T} / C_{p}$. The left-hand map will be an equivalence, and hence, we may compose an inverse of $r_{p}$ with the map $s_{p}$ to obtain the Frobenius map $\varphi_{p}$, well-defined up to contractible choice, in the infinity-category $\mathrm{Sp}^{B \mathbb{T}}$ of spectra with left $\mathbb{T}$-action. The topological Hochschild construction [12], Section 1.3.6] gives, more generally, a functor $\operatorname{THH}(\mathscr{C}, \mathscr{E}, \mathscr{W} ;-)$ from pointed spaces to symmetric spectra with left $\mathbb{T}$-action, whose value at $S^{0}$ agrees with $\operatorname{THH}(\mathscr{C}, \mathscr{E}, \mathscr{W})$. In order to define the geometric fixed point spectrum and the Tate spectrum, we fix the infinite dimensional complex $\mathbb{T}$-representation

$$
\mathscr{U}=\bigoplus_{k \in \mathbb{Z}, i \in \mathbb{N}} \mathbb{C}_{k, i}
$$

where $z \in \mathbb{T}$ acts on $\mathbb{C}_{k, i}$ by multiplication by $z^{k}$. We will write $V \subset \mathscr{U}$ to indicate that $V$ is a finite dimensional complex sub- $\mathbb{T}$-representation of $\mathscr{U}$. Now, the pointed space $\operatorname{THH}\left(\mathscr{C}, \mathscr{E}, \mathscr{W} ; S^{V}\right)_{r}$ has two left $\mathbb{T}$-actions, one coming from the cyclic structure and one induced by the left $\mathbb{T}$-action on $S^{V}$. If we give it the diagonal $\mathbb{T}$-action, then

$$
\left(\operatorname{THH}(\mathscr{C}, \mathscr{E}, \mathscr{W})^{\phi C_{p}}\right)_{r}=\operatorname{hocolim}_{V \subset \mathscr{U}, V^{C}=0}\left(\operatorname{THH}\left(\mathscr{C}, \mathscr{E}, \mathscr{W} ; S^{V}\right)_{r}^{C_{p}}\right)
$$

\footnotetext{
1 Recently, a fully homotopy invariant definition based on the Tate diagonal of [31. Section III.1] was given by Nikolaus [30].
} 
is the $r$ th space of the geometric fixed point spectrum. Similarly,

$$
\left(\operatorname{THH}(\mathscr{C}, \mathscr{E}, \mathscr{W})^{t C_{p}}\right)_{r}=\underset{V \subset \mathscr{U}, V^{C_{p}}=0}{\operatorname{hocolim}}\left(\operatorname{THH}\left(\mathscr{C}, \mathscr{E}, \mathscr{W} ; S^{V}\right)_{r}^{h C_{p}}\right)
$$

is the $r$ th space of the Tate spectrum, and the map $s_{p}$ is induced by the canonical map from fixed points to homotopy fixed points. The map $r_{p}$, in turn, is the map from the first homotopy colimit induced by $\mathbb{T}$-equivariant maps

$$
\rho_{p}^{*}\left(\operatorname{THH}\left(\mathscr{C}, \mathscr{E}, \mathscr{W} ; S^{V}\right)_{r}^{C_{p}}\right) \longrightarrow \operatorname{THH}\left(\mathscr{C}, \mathscr{E}, \mathscr{W} ; S^{0}\right)_{r}
$$

that exist in the Bökstedt model of topological Hochschild homology and are given by restricting a $C_{p}$-equivariant map to the induced map of $C_{p}$-fixed points. It is an equivalence by [31, Theorem III.4.7].

In general, one uses the Frobenius maps to define a number of spectra associated with a cyclotomic spectrum $X$. If $p$ is a prime number and $s \geqslant 1$ an integer, then the Tate orbit lemma, [31, Lemma I.2.1], implies that the canonical map

$$
X^{t C_{p^{s}}} \longrightarrow\left(X^{t C_{p}}\right)^{h\left(C_{p} s / C_{p}\right)}
$$

is an equivalence. Hence, by precomposing the inverse equivalence with the map of homotopy $C_{p^{s-1}}$-fixed points induced by $\varphi_{p}: X \rightarrow X^{t C_{p}}$, we obtain a map

$$
X^{h C_{p} s-1} \longrightarrow X^{t C_{p} s}
$$

and define

$$
\operatorname{TR}^{n}(X ; p)=X \times{ }_{X^{t} C_{p}} X^{h C_{p}} \times{ }_{X^{t C_{p^{2}}}} X^{h C_{p^{2}}} \times{ }_{X^{t C_{p}}} \cdots \times{ }_{X^{t C_{p^{n-1}}}} X^{h C_{p^{n-1}}},
$$

where $X^{h C_{p}^{s-1}} \rightarrow X^{t C_{p}^{s}}$ are the maps just defined, whereas $X^{t C_{p}^{s}} \leftarrow X^{h C_{p} s}$ are the canonical maps. Moreover, the restriction and Frobenius maps

$$
\operatorname{TR}^{n}(X ; p) \stackrel{R}{\underset{F}{\longrightarrow}} \operatorname{TR}^{n-1}(X ; p)
$$

are defined to be the projection onto the first $n-1$ factors and the composition of the projection onto the last $n-1$ factors and the map induced by the forgetful map $X^{h C_{p}} \rightarrow X$, respectively. Since the fiber of the restriction map agrees with the fiber of the canonical map $X^{h C_{p} p^{n-1}} \rightarrow X^{t C_{p^{n-1}}}$, we get the "fundamental" cofibration sequence

$$
X_{h C_{p^{n-1}}} \stackrel{N}{\longrightarrow} \mathrm{TR}^{n}(X ; p) \stackrel{R}{\longrightarrow} \mathrm{TR}^{n-1}(X ; p) .
$$

Finally, we define $\operatorname{TC}^{n}(X ; p)$ to be the homotopy equalizer of these two maps and define $\operatorname{TC}(X ; p)$ to be their homotopy limit as $n \geqslant 1$ varies. It is proved in [31, Theorem II.4.10] that if $X$ is $p$-complete, then the spectrum $\operatorname{TC}(X ; p)$ agrees canonically with the spectrum $\operatorname{TC}(X)$ considered in the introduction. 
If $X$ is the cyclotomic spectrum $\operatorname{THH}(\mathscr{C}, \mathscr{E}, \mathscr{W})$, then the Bökstedt-Dennis trace map lifts to a map of spectra called the cyclotomic trace map

$$
K(\mathscr{C}, \mathscr{E}, \mathscr{W}) \stackrel{\operatorname{tr}}{\longrightarrow} \mathrm{TC}(\mathscr{C}, \mathscr{E}, \mathscr{W}) .
$$

It is not clear from the definition that topological cyclic homology should be easier to understand than $K$-theory. We now explain why this is often the case.

As for $K$-theory, the additivity theorem [12, Proposition 2.0.4] implies that the adjunct structure maps

$$
\operatorname{THH}(\mathscr{C}, \mathscr{E}, \mathscr{W})_{r} \stackrel{\tilde{\sigma}_{r, s}}{\longrightarrow} \Omega^{s}\left(\operatorname{THH}(\mathscr{C}, \mathscr{E}, \mathscr{W})_{r+s}\right)
$$

are weak equivalences, for all integers $r \geqslant 1$ and $s \geqslant 0$. However, by contrast with $K$-theory, these maps are also weak equivalences, for $r=0$ and $s \geqslant 0$, if $\mathscr{E}$ is equal to the set of split-exact sequences in $\mathscr{C}$; see [12, Proposition 2.1.3]. Moreover, the inclusion of the 0 -skeleton in $N^{w}(\mathscr{C}, \mathscr{E}, \mathscr{W})$ induces a weak equivalence

$$
\mathrm{THH}(\mathscr{C}) \longrightarrow \operatorname{THH}(\mathscr{C}, \mathscr{E}, \mathscr{W})_{0},
$$

if $\mathscr{W}$ is equal to the subcategory of isomorphisms in $\mathscr{C}$.

Now let $A$ be a unital associative ring. By abuse of notation, we write $A$ also for the additive category with a single object $\emptyset$ whose ring of endomorphisms is $A$. There is an additive functor $i: A \rightarrow \mathscr{P}_{A}$ that to the unique object $\emptyset$ assigns $A$ considered as a left $A$-module under multiplication and that to $a \in \operatorname{End}_{A}(\emptyset)$ assigns the $A$-linear map $i(a) \in \operatorname{End}_{\mathscr{P}_{A}}(i(\emptyset))$ given by right multiplication by $a$. The induced map

$$
\operatorname{THH}(A) \stackrel{\mathrm{THH}(i)}{\longrightarrow} \operatorname{THH}\left(\mathscr{P}_{A}\right)
$$

is a weak equivalence by [12, Proposition 2.1.5]. The domain of this map is Bökstedt's original topological Hochschild space of the ring $A$, whose homotopy groups often are amenable to calculation. Hence, we conclude that Bökstedt's topological Hochschild homology groups and the homotopy groups of the topological Hochschild spectrum of the category $\mathscr{P}_{A}$ equipped with its canonical structure of exact category with weak equivalences agree, up to canonical natural isomorphism. We will abuse notation and write $\operatorname{THH}(A)$ also for the latter spectrum, and we write $\operatorname{TR}^{n}(A ; p), \mathrm{TC}^{n}(A ; p)$, etc. for the associated spectra defined above. The homotopy groups $\mathrm{THH}_{q}(A)$, however, are well-defined, up to canonical natural isomorphism.

We are now in a position to state and prove the analogue of Proposition 2.1 for topological Hochschild homology and its variants.

Proposition 2.2. Let $S$ be a complete discrete valuation ring with residue field $k_{S}$ and quotient field $K$ and let $R$ be an $S$-algebra. Assuming that, as a ring, $R$ is left regular, there is a canonical natural cofibration sequence of cyclotomic spectra

$$
\mathrm{THH}^{\prime}\left(R \otimes_{S} k_{S}\right) \stackrel{i_{*}}{\longrightarrow} \mathrm{THH}(R) \stackrel{j^{*}}{\longrightarrow} \mathrm{THH}\left(R \mid R \otimes_{S} K\right) \stackrel{\partial}{\longrightarrow} \Sigma \mathrm{THH}^{\prime}\left(R \otimes_{S} k_{S}\right),
$$

where the left-hand term and right-hand term denote the topological Hochschild spectra of $\mathscr{M}_{R \otimes_{S} k_{S}}$ and $\mathrm{Ch}^{b}\left(\mathscr{P}_{R}, \mathscr{M}_{R}, \mathscr{T}_{R}\right)$, respectively. The terms in the sequence 
have canonical $\mathrm{THH}(S)$-module structures and the morphisms in the sequence are $\mathrm{THH}(S)$-linear.

Proof. We repeat the proof of Proposition 2.1 mutatis mutandis. The analogues of Waldhausen's fibration theorem and Quillen's dévissage theorem hold and are proved in [20, Theorem 1.3.11] and [11, Theorem 1], respectively. However, the analogue of Waldhausen's approximation theorem, which is proved in [12, Proposition 2.3.2], requires a stronger hypothesis to be satisfied. In the situation at hand, it follows from [20, Lemma 1.5.3] and [8, Chapter XVII, Proposition 1.2] that the hypothesis is satisfied in all cases, with the exception that $\operatorname{THH}\left(R \mid R \otimes_{S} K\right)$ cannot be identified with $\operatorname{THH}\left(R \otimes_{S} K\right)$.

Addendum 2.3. Let $S$ be a complete discrete valuation ring with residue field $k_{S}$ and quotient field $K$ and let $R$ be an $S$-algebra. Assuming that, as a ring, $R$ is left regular, there is a commutative diagram of spectra

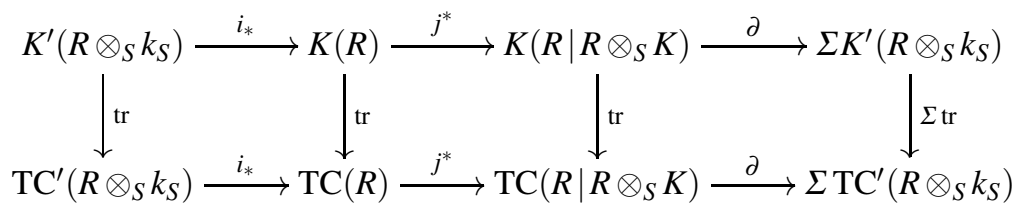

in which the rows are cofibration sequences.

Proof. The two sequences in the statement are obtained by applying respectively the $K$-theory functor and the topological cyclic homology functor to the same sequence of pointed exact categories with weak equivalences, so the diagram commutes by the naturality of the cyclotomic trace map. The sequences are cofibration sequences by Proposition 2.1 and Proposition 2.2, respectively.

Remark 2.4. If the ring $R \otimes_{S} k_{S}$ is artinian and if its quotient $\left(R \otimes_{S} k_{S}\right) / J$ by the radical is regular, then the morphisms

$$
K\left(\left(R \otimes_{S} k_{S}\right) / J\right) \longrightarrow K^{\prime}\left(\left(R \otimes_{S} k_{S}\right) / J\right) \longrightarrow K^{\prime}\left(R \otimes_{S} k_{S}\right)
$$

induced by the canonical inclusion functor and by the restriction-of-scalars functor are weak equivalences by [33, Corollary 2 of Theorem 3] and [33, Theorem 4]. The analogous statements for topological Hochschild homology and its variants hold by [11, Theorem 2] and [11, Theorem 1].

Theorem 2.5. With notation as in the introduction, the cyclotomic trace map induces isomorphisms of p-adic homotopy groups in degrees $j \geqslant 1$,

$$
K_{j}\left(D, \mathbb{Z}_{p}\right) \stackrel{\operatorname{tr}}{\longrightarrow} \operatorname{TC}_{j}\left(A \mid D, \mathbb{Z}_{p}\right) .
$$


Proof. The ring $A \otimes_{S} k_{S}$ is artinian and its quotient $k_{T}$ by the radical is regular. Hence, by Remark 2.4 Addendum 2.3 gives a commutative diagram of symmetric spectra

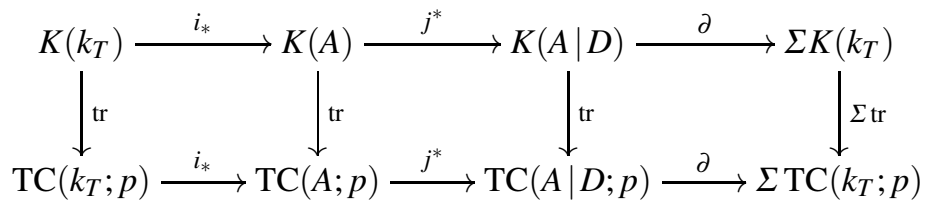

in which the rows are cofibration sequences. By [19, Theorem D], the first and second vertical morphisms from the left induce isomorphisms of $p$-adic homotopy groups in non-negative degrees, so the theorem follows from the five-lemma.

\section{Galois descent for topological cyclic homology}

In this section, we prove a rather general étale descent result for topological cyclic homology. The following result is well-known for commutative algebras.

Proposition 3.1. Let $S$ be a commutative ring, let $f: S \rightarrow T$ be an étale morphism of commutative rings, and let $A$ be any unital associative $S$-algebra, not necessarily commutative. In this case, the map induced by extension of scalars along $f: S \rightarrow T$,

$$
\mathrm{THH}_{j}(A) \otimes_{S} T \longrightarrow \mathrm{THH}_{j}\left(A \otimes_{S} T\right),
$$

is an isomorphism, for all integers $j$.

Proof. To fix notation, given a commutative $\operatorname{ring} S$, a unital associative $S$-algebra $R$, and an $S$-symmetric $R$ - $R$-bimodule $M$, one has the Hochschild homology $S$-modules $\mathrm{HH}_{j}(R / S ; M)$. We abbreviate and write $\mathrm{HH}_{j}(R / S)$ instead of $\mathrm{HH}_{j}(R / S ; M)$, if $M$ is $R$ considered as an $S$-symmetric $R$ - $R$-bimodule via left and right multiplication. We also abbreviate and write $\mathrm{HH}_{j}(R ; M)$ instead of $\mathrm{HH}_{j}(R / \mathbb{Z} ; M)$. If the $S$-algebra $R$ is commutative, then an $R$-module $N$ determines and is determined by a unique $R$ symmetric $R$ - $R$-bimodule $\tilde{N}$ with underlying $R$-module $N$. In this situation, we abuse notation and write $\mathrm{HH}_{j}(R / S ; N)$ instead of $\mathrm{HH}_{j}(R / S ; \tilde{N})$.

We recall from [15, Theorem 0.1$]$ that, for all integers $j$, the map

$$
\mathrm{HH}_{j}(S) \otimes_{S} T \longrightarrow \mathrm{HH}_{j}(T)
$$

induced by extension of scalars along $f: S \rightarrow T$ is an isomorphism. More generally, for every $S$-module $N$, the map induced by extension of scalars along $f: S \rightarrow T$,

$$
\mathrm{HH}_{j}(S ; N) \otimes_{S} T \longrightarrow \mathrm{HH}_{j}\left(T ; N \otimes_{S} T\right),
$$

is an isomorphism, for all integers $j$. Indeed, by choosing a simplicial resolution of the $S$-module $N$ by free $S$-modules, we are reduced to the case $N=S$. We conclude from [25, Theorem 3.1] that, similarly, the $T$-linear map

$$
\mathrm{THH}_{j}(S ; N) \otimes_{S} T \longrightarrow \mathrm{THH}_{j}\left(T ; N \otimes_{S} T\right)
$$


induced by extension of scalars along $f: S \rightarrow T$ is an isomorphism, for all integers $j$. It also follows from loc. cit. that there is a spectral sequence of $S$-modules

$$
E_{i, j}^{2}=\mathrm{HH}_{i}\left(A / S ; \mathrm{THH}_{j}(S ; A)\right) \Longrightarrow \mathrm{THH}_{i+j}(A),
$$

where the $S$-symmetric $A$-A-bimodule structure on $\operatorname{THH}_{j}(S ; A)$ is induced from left and right multiplication by $A$ on itself. We now extend scalars along the flat morphism $f: S \rightarrow T$ to obtain a spectral sequence of $T$-modules

$$
E_{s, t}^{2}=\mathrm{HH}_{i}\left(A / S ; \mathrm{THH}_{j}(S ; A)\right) \otimes_{S} T \Longrightarrow \mathrm{THH}_{i+j}(A) \otimes_{S} T,
$$

which we compare to the spectral sequence of $T$-modules

$$
E_{i, j}^{2}=\mathrm{HH}_{i}\left(A \otimes_{S} T / T ; \mathrm{THH}_{j}\left(T ; A \otimes_{S} T\right)\right) \Longrightarrow \mathrm{THH}_{i+j}\left(A \otimes_{S} T\right),
$$

which also is an instance of loc. cit. The map in the statement induces a map of spectral sequences which, on $E^{2}$-terms, is the composition of the isomorphism

$$
\mathrm{HH}_{i}\left(A / S ; \mathrm{THH}_{j}(S ; A)\right) \otimes_{S} T \longrightarrow \mathrm{HH}_{i}\left(A \otimes_{S} T / T ; \mathrm{THH}_{j}(S ; A) \otimes_{S} T\right)
$$

obtained by applying the exact functor $-\otimes_{S} T$ degreewise in the Hochschild complex and the isomorphism obtained by applying $\operatorname{HH}_{i}\left(A \otimes_{S} T / T ;-\right)$ to the isomorphism

$$
\mathrm{THH}_{j}(S ; A) \otimes_{S} T \longrightarrow \mathrm{THH}_{j}\left(T ; A \otimes_{S} T\right)
$$

of $T$-symmetric $A \otimes_{S} T-A \otimes_{S} T$-bimodules. This completes the proof.

Addendum 3.2. Let $S$ be a commutative ring, let $f: S \rightarrow T$ be an étale morphism of commutative rings, and let $A$ be any unital associative S-algebra, not necessarily commutative. In this case, the map induced by extension of scalars along $f: S \rightarrow T$,

$$
\operatorname{TR}_{j}^{n}(A ; p) \otimes_{W_{n}(S)} W_{n}(T) \longrightarrow \operatorname{TR}_{j}^{n}\left(A \otimes_{S} T ; p\right),
$$

is an isomorphism, for all prime numbers $p$ and integers $n \geqslant 1$ and $j$.

Proof. The proof is by induction on $n \geqslant 1$ with the case $n=1$ being already proved in Proposition 3.1. To prove the induction step, we use the following diagram

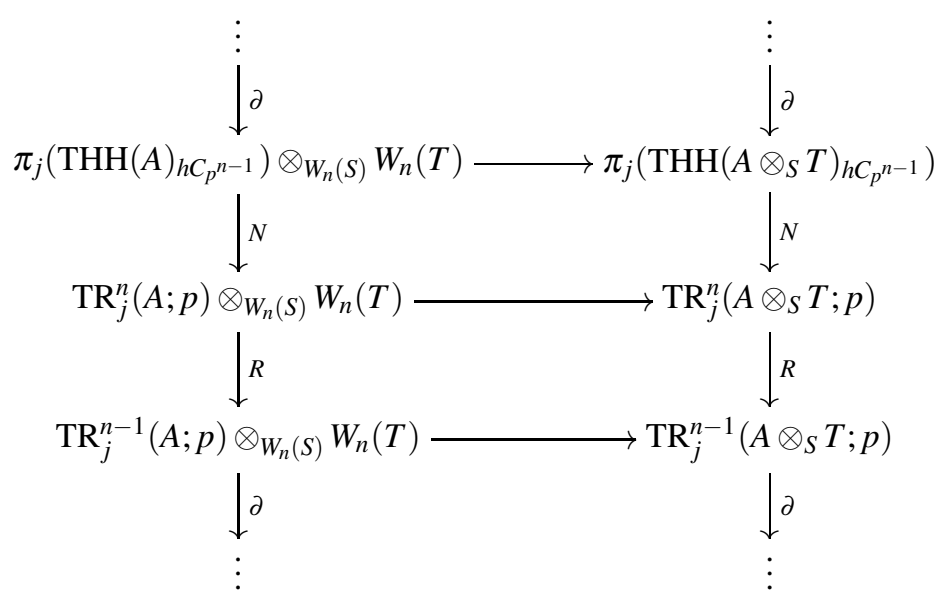


The right-hand column is the long exact sequence of homotopy groups induced by the "fundamental" cofibration sequence for the cyclotomic spectrum $\operatorname{THH}\left(A \otimes_{S} T\right)$. It is a sequence of $W_{n}(T)$-modules, by the argument in [19. pp. 71-72]. The lefthand column is obtained from the corresponding sequence for $\operatorname{THH}(A)$ by extension of scalars along $W_{n}(f): W_{n}(S) \rightarrow W_{n}(T)$ and it is exact, since the ring homomorphism $W_{n}(f): W_{n}(S) \rightarrow W_{n}(T)$ again is étale and hence flat by [4, Theorem B]. Moreover, the bottom groups $\mathrm{TR}_{j}^{n-1}(A ; p)$ and $\mathrm{TR}_{j}^{n-1}\left(A \otimes_{S} T ; p\right)$ are considered a $W_{n}(S)$-module and a $W_{n}(T)$-module, respectively, via the respective restriction maps. Therefore, by [5, Corollary 15.4], the bottom horizontal map agrees with the map

$$
\mathrm{TR}_{j}^{n-1}(A ; p) \otimes_{W_{n-1}(S)} W_{n-1}(T) \longrightarrow \mathrm{TR}_{j}^{n-1}\left(A \otimes_{S} T ; p\right)
$$

that we inductively assume to be an isomorphism. Hence, it will suffice to show that the top horizontal map is an isomorphism. The argument in [19. pp. 71-72] also shows that there is a natural spectral sequence of $W_{n}(S)$-modules

$$
E_{i, j}^{2}=H_{i}\left(C_{p^{n-1}},\left(F^{n-1}\right)_{*}\left(\mathrm{THH}_{j}(A)\right)\right) \Longrightarrow \pi_{i+j}\left(\mathrm{THH}(A)_{h C_{p^{n-1}}}\right)
$$

from the group homology of $C_{p^{n-1}}$ with coefficients in the $W_{n}(S)$-module obtained from the $S$-module $\mathrm{THH}_{j}(A)$ by restriction of scalars along $F^{n-1}: W_{n}(S) \rightarrow S$. By cobase-change along the flat ring homomorphism $W_{n}(f): W_{n}(S) \rightarrow W_{n}(T)$, this gives a spectral sequence of $W_{n}(T)$-modules converging to the domain of the top horizontal map in the diagram above. The target of this map, in turn, is the abutment of the spectral sequence of $W_{n}(T)$-modules

$$
E_{i, j}^{2}=H_{i}\left(C_{p^{n-1}},\left(F^{n-1}\right)_{*}\left(\mathrm{THH}_{j}\left(A \otimes_{S} T\right)\right)\right) \Longrightarrow \pi_{i+j}\left(\mathrm{THH}\left(A \otimes_{S} T\right)_{h C_{p^{n-1}}}\right),
$$

and the map in question induces a map from the former spectral sequence to the latter which, on $E^{2}$-terms, is the $W_{n}(T)$-linear map

$$
\left(F^{n-1}\right)_{*}\left(\mathrm{THH}_{j}(A)\right) \otimes_{W_{n}(S)} W_{n}(T) \longrightarrow\left(F^{n-1}\right)_{*}\left(\mathrm{THH}_{j}\left(A \otimes_{S} T\right)\right)
$$

induced by extension of scalars along $f: S \rightarrow T$. But [5, Corollary 15.4] shows that

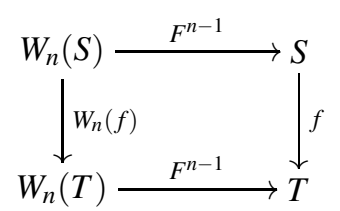

is a cocartesian diagram of commutative rings, so Proposition 3.1 implies that the this map is an isomorphism. This completes the proof.

A Galois extension of commutative rings is a pair $(f, \rho)$ of a faithfully étale ring homomorphism $f: S \rightarrow T$ and a left action $\rho: G \rightarrow \operatorname{Aut}_{S}(T)$ by a finite group $G$ on $T$ through $S$-algebra isomorphisms such that the ring homomorphism

$$
T \otimes_{S} T \stackrel{h}{\longrightarrow} \prod_{g \in G} T
$$


defined by $h\left(t_{1} \otimes t_{2}\right)=\left(g\left(t_{1}\right) t_{2}\right)_{g \in G}$ is an isomorphism. This notion is a special case of the general notion of a torsor in a topos [16, Chapitre III, Définition 1.4.1].

Corollary 3.3. Suppose that $\left(f: S \rightarrow T, \rho: G \rightarrow \operatorname{Aut}_{S}(T)\right)$ is a Galois extension of commutative rings and that $A$ is a unital associative $S$-algebra, not necessarily commutative. For all prime numbers $p$ and integers $n \geqslant 1$ and $j$, the $W_{n}(S)$-linear map induced by extension of scalars along $f: S \rightarrow T$,

$$
\operatorname{TR}_{j}^{n}(A ; p) \stackrel{f^{*}}{\longrightarrow} H^{0}\left(G, \operatorname{TR}_{j}^{n}\left(A \otimes_{S} T ; p\right)\right),
$$

is an isomorphism and the $W_{n}(S)$-modules $H^{i}\left(G, \operatorname{TR}_{j}^{n}\left(A \otimes_{S} T ; p\right)\right)$ with $i>0$ vanish.

Proof. Let $p$ be a prime number and let $n$ be a positive integer. We claim that

$$
\left(W_{n}(S) \stackrel{W_{n}(f)}{\longrightarrow} W_{n}(T), G \stackrel{W_{n} \circ \rho}{\longrightarrow} \operatorname{Aut}_{W_{n}(S)}\left(W_{n}(T)\right)\right)
$$

is a Galois extension of commutative rings. The ring homomorphism $W_{n}(f)$ is étale, by [4, Theorem B], and faithful, by op. cit., Proposition 6.9, and we now consider the commutative diagram

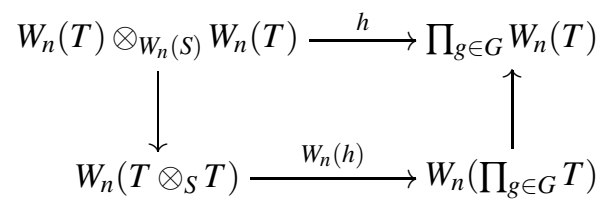

in which the vertical maps are the canonical maps. It is proved in op. cit., Corollary 9.4, that the left-hand vertical map is an isomorphism, and it follows immediately from the definition of Witt vectors that the right-hand vertical map is an isomorphism. Finally, by assumption, the lower horizontal map is an isomorphism, and hence, the top horizontal map is an isomorphism, as desired.

Finally, we abbreviate $M=\operatorname{TR}_{j}^{n}(A ; p), k=W_{n}(S)$, and $R=W_{n}(T)$, and consider the augmented cosimplicial $k$-module

$$
M \stackrel{\eta}{\longrightarrow} M \otimes_{k} R^{\otimes_{k}[-]} .
$$

Since $k \rightarrow R$ is faithfully flat, this map is a weak equivalence, by faithfully flat descent for modules. We also consider the augmented simplicial $k[G]$-module

$$
k[G]^{\otimes_{k}[-]} \otimes_{k} N \stackrel{\mu}{\longrightarrow} N,
$$

which is a weak equivalence for every $k[G]$-module $N$. Now, since $k \rightarrow R$ is Galois with group $G$, we have an isomorphism of cosimplicial $k$-modules

$$
M \otimes_{k} R^{\otimes_{k}[-]} \longrightarrow \operatorname{Hom}_{k[G]}\left(k[G]^{\otimes_{k}[-]}, M \otimes_{k} R\right) .
$$

Hence, we conclude that there is a canonical isomorphism

$$
M \longrightarrow R \operatorname{Hom}_{k[G]}\left(k, M \otimes_{k} R\right),
$$

in the derived category of $k$-modules. This proves the corollary. 


\section{Reduced trace isomorphisms}

We now prove the first part of Theorem $\mathrm{B}$, which is equivalent to the statement that there exists an element $\tilde{y} \in \mathrm{TC}_{0}\left(A \mid D, \mathbb{Z}_{p}\right)$ whose image $y_{1} \in \mathrm{THH}_{0}\left(A \mid D, \mathbb{Z}_{p}\right)$ freely generates $\mathrm{THH}_{*}\left(A \mid D, \mathbb{Z}_{p}\right)$ as a graded $\mathrm{THH}_{*}\left(S \mid K, \mathbb{Z}_{p}\right)$-module.

Lemma 4.1. Let $\pi \in A$ be a non-zero element. Conjugation by $\pi$ in $D$ defines an automorphism $\alpha: A \rightarrow A$ and the endomorphism of the cyclotomic spectrum

$$
\operatorname{THH}(A \mid D)=\operatorname{THH}\left(\mathrm{Ch}^{b}\left(\mathscr{P}_{A}, \mathscr{M}_{A}, \mathscr{T}_{A}\right)\right)
$$

induced by extension of scalars along $\alpha$ is homotopic to the identity.

Proof. Since $v_{D}\left(\pi x \pi^{-1}\right)=v_{D}(x)$, conjugation by $\pi$ in $D$ restricts to an automorphism $\alpha$ of $A$, as stated. Extension of scalars along $\alpha$ defines an exact functor

$$
\mathrm{Ch}^{b}\left(\mathscr{P}_{A}, \mathscr{M}_{A}, \mathscr{T}_{A}\right) \stackrel{\alpha^{*}}{\longrightarrow} \mathrm{Ch}^{b}\left(\mathscr{P}_{A}, \mathscr{M}_{A}, \mathscr{T}_{A}\right)
$$

and left multiplication by $\pi$ defines a natural transformation $h: \alpha^{*} \Rightarrow$ id. Indeed, if $P$ is in $\mathscr{P}_{A}$, then $\alpha^{*}(P)=A \otimes_{A} P$, where $A$ is considered as a right $A$-module via $\alpha$, and the map $h_{P}: A \otimes_{A} P \rightarrow P$, which maps $1 \otimes x$ to $\pi x$ is $A$-linear, since

$$
h_{P}(a \otimes x)=h_{P}\left(1 \otimes \pi^{-1} a \pi x\right)=\pi \pi^{-1} a \pi x=a \pi x=a h_{P}(x) .
$$

Since $\pi \in A$ is a non-zero-divisor, the natural transformation $h: \alpha^{*} \Rightarrow$ id is exact, and therefore, induces a homotopy through maps of cyclotomic spectra from the map induced by $\alpha^{*}$ to the identity map.

Addendum 4.2. The endomorphism of $\mathrm{THH}\left(A \otimes_{S} T \mid D \otimes_{K} L\right)$ induced by extension of scalars along $\alpha \otimes \mathrm{id}$ is homotopic to the identity, as a map of cyclotomic spectra.

Proof. The proof is entirely analogous to the proof of Lemma 4.1. That the natural transformation $h:(\alpha \otimes \mathrm{id})^{*} \Rightarrow \mathrm{id}$ is exact again uses that $\pi \otimes 1$ is a non-zero-divisor in the regular ring $A \otimes_{S} T$.

In particular, conjugation in $D$ by our chosen generator $\pi_{D} \in \mathfrak{m}_{D} \subset A$ defines an automorphism $\sigma: A \rightarrow A$. Moreover, since $\pi_{D}^{d} \in K$ is central, we see that the action of $G=\operatorname{Gal}(L / K)$ on $T / S$ extends to an action on $A / S$. This, in turn, induces an action of $G$ on the cyclotomic spectrum $\operatorname{THH}(A \mid D)$, and by Lemma 4.1, the action by the generator $\sigma \in G$ is homotopic to the identity map. It follows that $G$ acts trivially on the homotopy groups $\mathrm{THH}_{*}(A \mid D), \mathrm{TR}_{*}^{n}(A \mid D ; p), \mathrm{TC}_{*}(A \mid D)$, etc 2

Theorem 4.3. For all $n \geqslant 1$, the graded $\operatorname{TR}_{*}^{n}(S \mid K ; p)$-module $\operatorname{TR}_{*}^{n}(A \mid D ; p)$ is free of rank one on a canonical generator $y_{n}$ of degree zero.

\footnotetext{
2 By contrast, we show in Corollary 5.8 below that if $p$ divides $d$, then the $G$-action on the cyclotomic spectrum $\operatorname{THH}(A \mid D)$ is non-trivial.
} 
Proof. We consider the diagram of exact functors

$$
\begin{aligned}
\mathrm{Ch}^{b}\left(\mathscr{P}_{A}, \mathscr{M}_{A}, \mathscr{T}_{A}\right) \stackrel{f^{*}}{\longrightarrow} \mathrm{Ch}^{b}\left(\mathscr{P}_{A \otimes_{S} T}, \mathscr{M}_{A \otimes_{S} T}, \mathscr{T}_{A \otimes_{S} T}\right) \\
\operatorname{Trd}_{A \otimes_{S} T / T} \downarrow \mid \operatorname{Ird}_{A \otimes_{S} T / T} \\
\mathrm{Ch}^{b}\left(\mathscr{P}_{S}, \mathscr{M}_{S}, \mathscr{T}_{S}\right) \stackrel{f^{*}}{\longrightarrow} \mathrm{Ch}^{b}\left(\mathscr{P}_{T}, \mathscr{M}_{T}, \mathscr{T}_{T}\right),
\end{aligned}
$$

that we defined in the introduction. The group $G$ acts on $A$ and $T$, and we let it act diagonally on $A \otimes_{S} T$ and trivially on $S$. With respect to these actions, all functors in the diagram are $G$-equivariant, and hence, we obtain the following induced diagram of $\operatorname{THH}(S \mid K)$-modules in cyclotomic spectra with $G$-action,

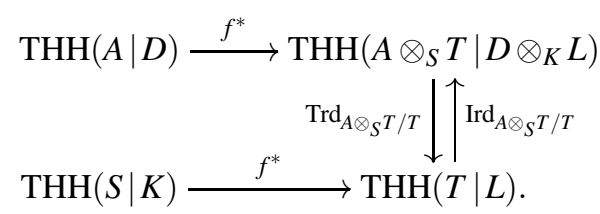

In this diagram, the right-hand vertical maps both are equivalences, since the counit and the unit of the adjunction $\left(\operatorname{Trd}_{A \otimes_{S} T / T}, \operatorname{Ird}_{A \otimes_{S} T / T}, \varepsilon, \eta\right)$ both are exact. While the $G$-action on the botton left-hand term is trivial, this is generally not true for the upper left-hand term. However, by Lemma 4.1, the action is trivial on homotopy groups, so we obtain the following induced diagram of graded $\mathrm{TR}_{*}^{n}(S \mid K ; p)$-modules,

$$
\begin{aligned}
\operatorname{TR}_{*}^{n}(A \mid D ; p) \stackrel{f^{*}}{\longrightarrow} H^{0}\left(G, \operatorname{TR}_{*}^{n}\left(A \otimes_{S} T \mid D \otimes_{K} L ; p\right)\right) \\
\operatorname{Trd}_{A \otimes_{S} T / T} \downarrow \uparrow \operatorname{Ird}_{A \otimes_{S} T / T} \\
\operatorname{TR}_{*}^{n}(S \mid K ; p) \stackrel{f^{*}}{\longrightarrow} H^{0}\left(G, \operatorname{TR}_{*}^{n}(T \mid L ; p)\right) .
\end{aligned}
$$

In this diagram, the horizontal maps are isomorphisms by Corollary 3.3 , and the vertical maps are isomorphisms by what was just said. Moreover, by Skolem-Noether, all $K$-algebra homomorphisms $L \rightarrow D$ are conjugate in $D$, and therefore, it follows from Addendum 4.2 that the vertical maps are independent of the choice of maximal unramified subfield $L \subset D$. Hence, we obtain canonical inverse isomorphisms

$$
\mathrm{TR}_{*}^{n}(A \mid D ; p) \underset{\operatorname{Ird}_{A / S}}{\stackrel{\operatorname{Trd}_{A / S}}{\rightleftarrows}} \operatorname{TR}_{*}^{n}(S \mid K ; p)
$$

given by the maps making the respective square diagrams commute. Equivalently, the graded $\mathrm{TR}_{*}^{n}(S \mid K ; p)$-module $\operatorname{TR}_{*}^{n}(A \mid D ; p)$ is free on the single canonical generator $y_{n}=\operatorname{Ird}_{A / S}(1) \in \operatorname{TR}_{0}^{n}(A \mid D ; p)$, as stated.

Next, we wish to prove the analogous result for the $p$-adic homotopy groups. In general, the $p$-adic homotopy groups of a spectrum $X$ are defined to the homotopy groups of its $p$-completion, and by [7, Proposition 2.5] there is an exact sequence

$$
0 \rightarrow \operatorname{Ext}_{\mathbb{Z}}^{1}\left(\mathbb{Q}_{p} / \mathbb{Z}_{p}, \pi_{j}(X)\right) \rightarrow \pi_{j}\left(X, \mathbb{Z}_{p}\right) \rightarrow \operatorname{Hom}_{\mathbb{Z}}\left(\mathbb{Q}_{p} / \mathbb{Z}_{p}, \pi_{j-1}(X)\right) \rightarrow 0
$$

that relates the homotopy groups of $X$ and the $p$-adic homotopy groups of $X$. 
Definition 4.4. An abelian group $M$ is $p$-controlled if it is a direct sum of a uniquely divisible $M_{\mathrm{div}}$ and a group $M_{\mathrm{tor}}$ annihilated by some power of $p$.

Lemma 4.5. If $M$ and $N$ are $p$-controlled, then every extension of $N$ by $M$ and the kernel and cokernel of every homomorphism $M \rightarrow N$ are also p-controlled. If $M$ is p-controlled, then $\operatorname{Hom}_{\mathbb{Z}}\left(M, \mathbb{Z}_{p}\right)$ and $\operatorname{Hom}_{\mathbb{Z}}\left(\mathbb{Q}_{p} / \mathbb{Z}_{p}, M\right)$ both vanish.

Proof. The only statement that needs proof is that the full subcategory of the abelian category of abelian groups is closed under extensions. So we let

$$
0 \longrightarrow M \longrightarrow P \longrightarrow N
$$

be an exact sequence of abelian groups, where $M$ and $N$ are $p$-controlled, and wish to show that $P$ is $p$-controlled. As uniquely divisible groups are $\mathbb{Z}$-injective, $P$ is isomorphic to $M_{\mathrm{div}} \oplus P / M_{\mathrm{div}}$, so replacing $M$ and $P$ by $M_{\mathrm{tor}}$ and $P / M_{\mathrm{div}}$, respectively, we assume that $M=M_{\text {tor }}$. Let $P^{\text {tor }}$ and $P^{\text {div }}$ be the inverse images of $N_{\text {tor }}$ and $N_{\text {div }}$ in $P$, respectively. It suffices to prove that $P^{\text {tor }}$ and $P^{\text {div }}$ are $p$-controlled, so we may reduce to the two cases $N=N_{\text {tor }}$ and $N=N_{\text {div }}$. The first is trivial, and for the second, it suffices to prove that $\operatorname{Ext}_{\mathbb{Z}}^{1}(N, M)=0$. But this is clear, since this group is both $p$-divisible and killed by a power of $p$.

Proposition 4.6. The group $\operatorname{TR}_{j}^{n}(S \mid K ; p)$ is $p$-controlled, for all $n \geqslant 1$ and $j \geqslant 1$.

Proof. The groups $\operatorname{TR}_{j}^{n}\left(k_{S} ; p\right)$ are finite $p$-groups for all integers $n \geqslant 1$ and $j$. Hence, by Proposition 2.2 and Lemma 4.5, it suffices to show that $\operatorname{TR}_{j}^{n}(S ; p)$ is $p$-controlled, for all $n \geqslant 1$ and $j \geqslant 1$. If $S$ is of equal characteristic, then the groups $\operatorname{TR}_{j}^{n}(S ; p)$ are annihilated by $p^{n}$. So we assume that $S$ is of mixed characteristic and proceed by induction on $n \geqslant 1$. In the case $n=1$, we use the spectral sequence

$$
E_{i, j}^{2}=\mathrm{HH}_{i}\left(S / \mathbb{Z}_{p}, \mathrm{THH}_{j}\left(\mathbb{Z}_{p}\right) \otimes_{\mathbb{Z}_{p}} S\right) \Rightarrow \mathrm{THH}_{i+j}(S)
$$

from [25, Corollary 3.3]. If $j \geqslant 1$, then $\mathrm{THH}_{j}\left(\mathbb{Z}_{p}\right)$ is $p$-controlled, by Theorem 2.2 and Example 3.4 of [24], and hence, so are the groups $E_{i, j}^{2}$, since $\mathrm{HH}_{i}\left(S / \mathbb{Z}_{p}, M\right)$ is $M$, for $i=0$, and is annihilated by a fixed power of $p$, for $i>0$. This also shows that $E_{i, 0}^{2}$ is $p$-controlled, for $i>0$. Therefore, Lemma 4.5 and the spectral sequence shows that $\mathrm{THH}_{j}(S)$ is $p$-controlled.

To prove the induction step, we use the "fundamental" cofibration

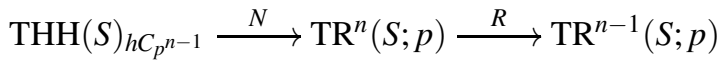

and the spectral sequence

$$
E_{i, j}^{2}=H_{i}\left(C_{p^{n-1}}, \mathrm{THH}_{j}(S)\right) \Rightarrow \pi_{i+j}\left(\mathrm{THH}(S)_{h C_{p^{n-1}}}\right) .
$$

By the case $n=1$, we conclude that $E_{i, j}^{2}$ is $p$-controlled, if $i>0$ or $j>0$, and hence, Lemma 4.5 shows that $\pi_{j}\left(\mathrm{THH}(S)_{h C_{p} n-1}\right)$ is $p$-controlled, for $j>0$. The induction step now follows from Lemma 4.5, since the boundary map

$$
\mathrm{TR}_{1}^{n-1}(S ; p) \stackrel{\partial}{\longrightarrow} \pi_{0}\left(\mathrm{THH}(S)_{h C_{p}{ }^{n-1}}\right)
$$

is zero for every commutative ring. 
Addendum 4.7. The graded $\mathrm{TR}_{*}^{n}\left(S \mid K ; p, \mathbb{Z}_{p}\right)$-module $\mathrm{TR}_{*}^{n}\left(A \mid D ; p, \mathbb{Z}_{p}\right)$ is free of rank one with canonical generator $y_{n} \in \operatorname{TR}_{0}^{n}\left(A \mid D ; p, \mathbb{Z}_{p}\right)$, for all $n \geqslant 1$.

Proof. If $S$ is of equal characteristic, then $\operatorname{TR}^{n}(S \mid K ; p)$ and $\mathrm{TR}^{n}(A \mid D ; p)$ are already $p$-complete, so there is nothing further to prove. If $S$ is of mixed characteristic, then by Proposition 4.6 and Lemma 4.5 , the horizontal maps in the diagram

$$
\begin{array}{cc}
\operatorname{Ext}_{\mathbb{Z}}^{1}\left(\mathbb{Q}_{p} / \mathbb{Z}_{p}, \operatorname{TR}_{*}^{n}(A \mid D ; p)\right) & \left.\operatorname{TR}_{*}^{n}\left(A \mid D ; p, \mathbb{Z}_{p}\right)\right) \\
\operatorname{Trd}_{A / S} \mid \uparrow \operatorname{Ird}_{A / S} & \operatorname{Trd}_{A / S} \mid \uparrow \operatorname{Ird}_{A / S} \\
\operatorname{Ext}_{\mathbb{Z}}^{1}\left(\mathbb{Q}_{p} / \mathbb{Z}_{p}, \operatorname{TR}_{*}^{n}(S \mid K ; p)\right) \longrightarrow & \left.\operatorname{TR}_{*}^{n}\left(S \mid K ; p, \mathbb{Z}_{p}\right)\right)
\end{array}
$$

of graded $\mathrm{TR}_{*}^{n}\left(S \mid K ; p, \mathbb{Z}_{p}\right)$-modules are isomorphisms, and we define the right-hand vertical maps to be the unique maps that make the respective square diagrams commute. Finally, by Theorem 4.3 , the left-hand vertical maps are mutally inverse isomorphisms, and hence, so are the right-hand vertical maps.

Lemma 4.8. For all $j \geqslant 1$, the limit systems

$$
\begin{aligned}
& \cdots \stackrel{R}{\longrightarrow} \operatorname{TR}_{j}^{n}\left(S ; p, \mathbb{Z}_{p}\right) \stackrel{R}{\longrightarrow} \cdots \stackrel{R}{\longrightarrow} \operatorname{TR}_{j}^{2}\left(S ; p, \mathbb{Z}_{p}\right) \stackrel{R}{\longrightarrow} \operatorname{TR}_{j}^{1}\left(S ; p, \mathbb{Z}_{p}\right) \\
& \cdots \stackrel{F}{\longrightarrow} \operatorname{TR}_{j}^{n}\left(S ; p, \mathbb{Z}_{p}\right) \stackrel{F}{\longrightarrow} \cdots \stackrel{F}{\longrightarrow} \operatorname{TR}_{j}^{2}\left(S ; p, \mathbb{Z}_{p}\right) \stackrel{F}{\longrightarrow} \operatorname{TR}_{j}^{1}\left(S ; p, \mathbb{Z}_{p}\right)
\end{aligned}
$$

both satisfy the Mittag-Leffler condition.

Proof. If $S$ is of mixed characteristic, then the groups $\operatorname{TR}_{j}^{n}\left(S ; p, \mathbb{Z}_{p}\right)$ with $j \geqslant 1$ are all finite $p$-groups. Indeed, the case $n=1$ is proved in [26], and the general case follows by an inductive argument similar to the proof of Proposition 4.6 above. In particular, the limit systems satisfy the Mittag-Leffler condition. If $S$ is of equal characteristic, then it follows from [17, Theorem B] that the canonical map

$$
W_{n} \Omega_{S}^{*} \otimes \mathrm{TR}_{*}^{n}\left(\mathbb{F}_{p} ; p\right) \longrightarrow \mathrm{TR}_{*}^{n}(S ; p)
$$

is an isomorphism. Indeed, the ring $\left.S=k_{S}[t]\right]$ is a regular $k_{S}$-algebra, and hence, is a filtered colimit of smooth $k_{S}$-algebras by [32]. Moreover, by Bökstedt periodicity, we have $\mathrm{TR}_{*}^{n}\left(\mathbb{F}_{p} ; p\right)=\mathbb{Z} / p^{n}\left[x_{n}\right]$, where $x_{n}$ has degree 2 and may be chosen such that $R\left(x_{n}\right)=p x_{n-1}$ and $F\left(x_{n}\right)=x_{n-1}$. The structure of the de Rham-Witt groups was determined in [14, Theorem B]. It shows in particular that

$$
\begin{aligned}
& \operatorname{im}\left(\lim _{m, R} \operatorname{TR}_{j}^{m}(S ; p) \stackrel{\mathrm{pr}}{\longrightarrow} \operatorname{TR}_{j}^{n}(S ; p)\right)=\operatorname{im}\left(\operatorname{TR}_{j}^{2 n}(S ; p) \stackrel{R^{n}}{\longrightarrow} \operatorname{TR}_{j}^{n}(S ; p)\right), \\
& \operatorname{im}\left(\lim _{m, F} \operatorname{TR}_{j}^{m}(S ; p) \stackrel{\mathrm{pr}}{\longrightarrow} \operatorname{TR}_{j}^{n}(S ; p)\right)=\operatorname{im}\left(\operatorname{TR}_{j}^{2 n}(S ; p) \stackrel{F^{n}}{\longrightarrow} \operatorname{TR}_{j}^{n}(S ; p)\right),
\end{aligned}
$$

whence the lemma.

Theorem 4.9. The graded $\operatorname{TR}_{*}\left(S \mid K ; p, \mathbb{Z}_{p}\right)$-module $\operatorname{TR}_{*}\left(A \mid D ; p, \mathbb{Z}_{p}\right)$ is free on a canonical generator $y$. The graded $\mathrm{TF}_{*}\left(S \mid K ; p, \mathbb{Z}_{p}\right)$-module $\mathrm{TF}_{*}\left(A \mid D ; p, \mathbb{Z}_{p}\right)$ is free on a canonical generator $y$. 
Proof. We prove the second statement; the first statement is proved analogously. By Theorem 4.3, the graded $\operatorname{TR}_{*}^{n}\left(S \mid K ; p, \mathbb{Z}_{p}\right)$-module $\operatorname{TR}_{*}^{n}\left(A \mid D ; p, \mathbb{Z}_{p}\right)$ is free on the canonical generator $y_{n}=\operatorname{Ird}_{A / S}(1) \in \operatorname{TR}_{0}^{n}\left(A \mid D ; p, \mathbb{Z}_{p}\right)$. Moreover, we have

$$
F\left(y_{n}\right)=F\left(\operatorname{Ird}_{A / S}(1)\right)=\operatorname{Ird}_{A / S}(F(1))=\operatorname{Ird}_{A / S}(1)=y_{n-1} .
$$

Indeed, the second identity holds, since the maps

$$
\begin{aligned}
& \operatorname{THH}(A \mid D) \stackrel{f^{*}}{\longrightarrow} \operatorname{THH}\left(A \otimes_{S} T \mid D \otimes_{K} L\right) \\
& \operatorname{THH}(S \mid K) \stackrel{f^{*}}{\operatorname{Trd}_{A \otimes_{S} T / T} \downarrow \mid \operatorname{Ird}_{A \otimes_{S} T / T}} \longrightarrow \operatorname{THH}(T \mid L) .
\end{aligned}
$$

that we used to define the isomorphism $\operatorname{Ird}_{A / S}$ are maps of cyclotomic spectra, and the third identity holds, since $F$ is a ring homomorphism. Finally, we claim that the canonical maps

$$
\begin{aligned}
& \mathrm{TF}_{j}\left(A \mid D ; p, \mathbb{Z}_{p}\right) \longrightarrow \lim _{n, F} \operatorname{TR}_{j}^{n}\left(A \mid D ; p, \mathbb{Z}_{p}\right) \\
& \mathrm{TF}_{j}\left(S \mid K ; p, \mathbb{Z}_{p}\right) \longrightarrow \lim _{n, F} \operatorname{TR}_{j}^{n}\left(S \mid K ; p, \mathbb{Z}_{p}\right)
\end{aligned}
$$

are isomorphisms, or equivalently, that the corresponding derived limits vanish. In the case $j \geqslant 1$, this follows from Lemma 4.8 and in the case $j=0$, it follows from the fact that $\operatorname{TR}_{0}^{n}\left(S \mid K ; p, \mathbb{Z}_{p}\right)=W_{n}(S)$ admits a compact topology with respect to which the map $F$ is continuous. So the theorem follows with $y \in \mathrm{TF}_{0}\left(A \mid D ; p, \mathbb{Z}_{p}\right)$ the unique class with image $y_{n} \in \operatorname{TR}_{0}^{n}\left(A \mid D ; p, \mathbb{Z}_{p}\right)$ for all $n \geqslant 1$.

Proof of Theorem $B$ (1). We recall that for a cyclotomic spectrum $X$, its topological cyclic homology is equivalently given as the homotopy equalizer

$$
\mathrm{TC}(X ; p) \stackrel{i}{\longrightarrow} \mathrm{TF}(X ; p) \stackrel{\varphi^{-1}}{\longrightarrow} \mathrm{id}(X ; p),
$$

where the inverse Frobenius $\varphi^{-1}$ is defined to be the composition

$$
\operatorname{holim}_{n, F} \mathrm{TR}^{n}(X ; p) \stackrel{\operatorname{res}_{\sigma}}{\longrightarrow} \operatorname{holim}_{n, F} \mathrm{TR}^{n+1}(X ; p) \stackrel{R}{\longrightarrow} \operatorname{holim}_{n, F} \operatorname{TR}^{n}(X ; p)
$$

of the restriction along the successor functor followed by the map of limits induced by the restriction maps. Hence, in the induced diagram

$$
\mathrm{TC}_{0}(X ; p) \stackrel{i}{\longrightarrow} \mathrm{TF}_{0}(X ; p) \stackrel{\varphi^{-1}}{\longrightarrow} \mathrm{TF}_{0}(X ; p)
$$

the map $i$ surjects onto the equalizer of maps $\varphi^{-1}$ and id. Now, if $X$ is the cyclotomic spectrum $\operatorname{THH}\left(A \mid D, \mathbb{Z}_{p}\right)$, then the element $y \in \mathrm{TF}_{0}\left(A \mid D ; p, \mathbb{Z}_{p}\right)$ satisfies $\varphi^{-1}(y)=y$, 
because $R\left(y_{n}\right)=y_{n-1}$. Hence, there exists $\tilde{y} \in \mathrm{TC}_{0}\left(A \mid D, \mathbb{Z}_{p}\right)$ such that $i(\tilde{y})=y$. The class $\tilde{y}$ defines a component in the mapping space

$$
\begin{aligned}
& \operatorname{Map}_{\operatorname{Mod}_{\mathrm{THH}\left(S \mid K, \mathbb{Z}_{p}\right)}\left(\operatorname{CycSp}_{p}\right)}\left(\operatorname{THH}\left(S \mid K, \mathbb{Z}_{p}\right), \operatorname{THH}\left(A \mid D, \mathbb{Z}_{p}\right)\right) \\
& \simeq \operatorname{Map}_{\mathrm{CycSp}_{p}}\left(\mathbb{S}_{p}, \operatorname{THH}\left(A \mid D, \mathbb{Z}_{p}\right)\right) \simeq \Omega^{\infty} \operatorname{TC}\left(A \mid D, \mathbb{Z}_{p}\right),
\end{aligned}
$$

and every point in this component induces the isomorphism

$$
\mathrm{THH}_{*}\left(S \mid K, \mathbb{Z}_{p}\right) \stackrel{\operatorname{Ird}_{A / S}}{\longrightarrow} \mathrm{THH}_{*}\left(A \mid D, \mathbb{Z}_{p}\right)
$$

on the level of homotopy groups. Indeed, the class $\tilde{y} \in \mathrm{TC}_{0}\left(A \mid D, \mathbb{Z}_{p}\right)$ maps to the class $y_{1} \in \mathrm{THH}_{0}\left(A \mid D, \mathbb{Z}_{p}\right)$ and $\operatorname{Ird}_{A / S}$ is given by multiplication by $y_{1}$. This shows that every point in the component $\tilde{y}$ of the mapping space defines an equivalence of $\mathrm{THH}\left(S \mid K, \mathbb{Z}_{p}\right)$-modules in cyclotomic spectra,

$$
\operatorname{THH}\left(S \mid K, \mathbb{Z}_{p}\right) \stackrel{\operatorname{Ird}_{A / S}}{\longrightarrow} \operatorname{THH}\left(A \mid D, \mathbb{Z}_{p}\right)
$$

whose inverse $\operatorname{Trd}_{A / S}$ therefore also is an equivalence of $\operatorname{THH}\left(S \mid K, \mathbb{Z}_{p}\right)$-modules in cyclotomic spectra. This proves part (1) of the Theorem $B$

\section{Comparison with the trace map}

The equivalence of cyclotomic spectra in Theorem $\mathrm{B}$ (1) shows, in particular, that the graded $\mathrm{TC}_{*}^{-}\left(S \mid K, \mathbb{Z}_{p}\right)$-module $\mathrm{TC}_{*}^{-}\left(A \mid D, \mathbb{Z}_{p}\right)$ and the graded $\mathrm{TP}_{*}\left(S \mid K, \mathbb{Z}_{p}\right)$-module $\mathrm{TP}_{*}\left(A \mid D, \mathbb{Z}_{p}\right)$ both are free of rank one generated by the image under

$$
\mathrm{TC}_{*}\left(A \mid D, \mathbb{Z}_{p}\right) \stackrel{i}{\longrightarrow} \mathrm{TC}_{*}^{-}\left(A \mid D, \mathbb{Z}_{p}\right) \stackrel{\varphi}{\underset{\mathrm{can}}{\longrightarrow}} \mathrm{TP}_{*}\left(A \mid D, \mathbb{Z}_{p}\right)
$$

of the generator $\tilde{y} \in \mathrm{TC}_{0}\left(A \mid D, \mathbb{Z}_{p}\right)$. We first use results from [14] and [20] to prove structural results about these groups.

Lemma 5.1. The Frobenius map

$$
\operatorname{THH}\left(S \mid K, \mathbb{Z}_{p}\right) \stackrel{\varphi}{\longrightarrow} \mathrm{THH}\left(S \mid K, \mathbb{Z}_{p}\right)^{t C_{p}}
$$

induces an equivalence of connective covers.

Proof. If $S$ is of mixed characteristic, then this is proved in [20, Theorem 5.4.3]. We therefore assume that $S=k_{S}[[\pi]]$ is of equal characteristic. Since the Frobenius $\varphi: \operatorname{THH}\left(k_{S}\right) \rightarrow \operatorname{THH}\left(k_{S}\right)^{t C_{p}}$ induces an equivalence of $(-1)$-connective covers, it suffices to show that the Frobenius $\varphi: \mathrm{THH}(S) \rightarrow \mathrm{THH}(S)^{t} C_{p}$ induces an equivalence of connective covers. Now, by [17, Theorem B] and [32], the canonical map

$$
\Omega_{S}^{*} \otimes \mathrm{THH}_{*}\left(\mathbb{F}_{p}\right) \longrightarrow \mathrm{THH}_{*}(S)
$$


is an isomorphism. Since $k_{S}$ is perfect, $\Omega_{S}^{*}$ is an exterior algebra over $S$ on a generator $d \pi$ of degree 1 , and $\mathrm{THH}_{*}\left(\mathbb{F}_{p}\right)$ is a polynomial algebra over $\mathbb{F}_{p}$ on a generator $x$ of degree 2 by Bökstedt periodicity. Hence, in the Tate spectral sequence

$$
E_{i, j}^{2}=\hat{H}^{-i}\left(C_{p}, \mathrm{THH}_{j}(S)\right) \Rightarrow \pi_{i+j}\left(\operatorname{THH}(S)^{t C_{p}}\right),
$$

we have $E^{2}=S \otimes \Lambda\{u, d \pi\} \otimes \mathbb{F}_{p}\left[t^{ \pm 1}, x\right]$ with $\operatorname{deg}(u)=(-1,0), \operatorname{deg}(d \pi)=(0,1)$, $\operatorname{deg}(t)=(-2,0)$, and $\operatorname{deg}(x)=(0,2)$. The $d^{2}$-differential is a derivation, which is automatically $S^{p}$-linear, and is given by $d^{2}(\pi)=t d \pi$, so the $E^{3}$-term takes the form

$$
E^{3}=S^{p} \otimes \Lambda\left\{u, \pi^{p-1} d \pi\right\} \otimes \mathbb{F}_{p}\left[t^{ \pm 1}, x\right] .
$$

The Frobenius $\varphi$ maps the class $a \in S$ (resp. $d \pi$, resp. $x$ ) to the class represented in the spectral sequence by $a^{p} \in S^{p}$ (resp. by $\pi^{p-1} d \pi$, resp. by $t^{-1}$ ), which therefore is an infinite cycle. Moreover, comparing with the spectral sequence for $\mathrm{THH}\left(\mathbb{F}_{p}\right)$, we see that also $x$ is an infinite cycle and that, up to a unit in $\mathbb{F}_{p}$,

$$
d^{3}(u)=t^{2} x .
$$

Hence, all further differentials in the spectral sequence are zero, and

$$
E^{\infty}=S^{p} \otimes \Lambda\left\{\pi^{p-1} d \pi\right\} \otimes \mathbb{F}_{p}\left[t^{ \pm 1}\right] .
$$

In particular, the Frobenius $\varphi: \operatorname{THH}(S) \rightarrow \operatorname{THH}(S)^{t C_{p}}$ induces an isomorphism of homotopy groups in degrees $j \geqslant 0$, as desired.

Corollary 5.2. The Frobenius map induces an equivalence of connective covers

$$
\mathrm{TF}\left(S \mid K ; p, \mathbb{Z}_{p}\right) \longrightarrow \mathrm{TC}^{-}\left(S \mid K, \mathbb{Z}_{p}\right) .
$$

Proof. For every cyclotomic spectrum $X$, we have the canonical projection

$$
\operatorname{TR}^{n}(X ; p)=X \times{ }_{X^{t} C_{p}} X^{h C_{p}} \times{ }_{X^{t C_{p}}} \cdots \times{ }_{X^{t C_{p}}{ }^{n-1}} X^{h C_{p^{n-1}}} \stackrel{\mathrm{pr}_{n}}{\longrightarrow} X^{h C_{p^{n-1}}}
$$

and there are commutative diagrams

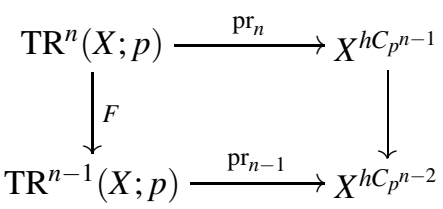

in which the horizontal maps are the canonical projections and the right-hand vertical maps are the map induced by the subgroup inclusions $C_{p^{n-2}} \subset C_{p^{n-1}}$. Hence, the canonical projections induce a map of homotopy limits

$$
\mathrm{TF}(X ; p)=\operatorname{holim}_{n, F} \mathrm{TR}^{n}(X ; p) \longrightarrow \operatorname{holim}_{n} X^{h C_{p^{n-1}}} .
$$

Moreover, if $X$ is $p$-complete, then the map

$$
\operatorname{holim}_{n} X^{h C_{p^{n-1}} \longleftarrow} X^{h \mathbb{T}}=\mathrm{TC}^{-}(X),
$$


induced by the subgroup inclusions $C_{p^{n-1}} \subset \mathbb{T}$ is an equivalence, so we get a map

$$
\mathrm{TF}(X ; p) \longrightarrow \mathrm{TC}^{-}(X) \text {. }
$$

As pointed out in the proof of [22, Theorem 3], it is clear from this construction that if the Frobenius $\varphi: X \rightarrow X^{t C_{p}}$ induces an isomorphism of homotopy groups in degrees $j \geqslant d$, then so do the maps $\operatorname{pr}_{n}$ and map above. So by taking $X$ to be the $p$-complete cyclotomic spectrum $\operatorname{THH}\left(S \mid K, \mathbb{Z}_{p}\right)$, the corollary follows from Lemma 5.1

Lemma 5.3. The canonical projection $i: S \rightarrow k_{S}$ induces an isomorphism

$$
\lim _{n, F} W_{n}(S) \longrightarrow \lim _{n, F} W_{n}\left(k_{S}\right) .
$$

Proof. Let $\mathfrak{m}_{S} \subset S$ be the maximal ideal. The injectivity and the surjectivity of the map in the statement are equivalent to the vanishing of the $\operatorname{limit}_{n, F} \lim _{n}\left(\mathfrak{m}_{S}\right)$ and the derived limit $R^{1} \lim _{n, F} W_{n}\left(\mathfrak{m}_{S}\right)=0$, respectively. We recall that the Witt vector Frobenius $F: W_{n}(S) \rightarrow W_{n-1}(S)$ satisfies $F(a)=R(a)^{p}$ modulo $W_{n-1}(p S)$; see for example [18, Lemma 1.8]. It follows that $F\left(W_{n}\left(\mathfrak{m}_{S}^{m}\right)\right) \subset W_{n-1}\left(\mathfrak{m}_{S}^{m+1}\right)$, for all $m>0$, which shows that the limit vanishes. The derived limit vanishes, since $W_{n}\left(\mathfrak{m}_{S}\right)$ has a compact topology for which $F: W_{n}\left(\mathfrak{m}_{S}\right) \rightarrow W_{n-1}\left(\mathfrak{m}_{S}\right)$ is continuous.

Since $k_{S}$ is perfect, we further identify the common ring in Lemma 5.3 with the ring of Witt vectors $W\left(k_{s}\right)$ via the isomorphism

$$
\lim _{n, F} W_{n}\left(k_{S}\right) \longrightarrow \lim _{n, R} W_{n}\left(k_{S}\right)=W\left(k_{S}\right)
$$

that at level $n$ is given by the map $W_{n}\left(k_{S}\right) \rightarrow W_{n}\left(k_{S}\right)$ induced by $\varphi^{n}: k_{S} \rightarrow k_{S}$. It is an isomorphism, since $k_{S}$ is perfect. In particular, the ring

$$
\mathrm{TF}_{0}\left(S \mid K ; p, \mathbb{Z}_{p}\right)=\lim _{n, F} W_{n}(S)
$$

is an integral domain.

Theorem 5.4. For every even integer $j=2 k>0$, the map

$$
\mathrm{TC}_{j}^{-}\left(S \mid K, \mathbb{Z}_{p}\right) \stackrel{\varphi-\text { can }}{\longrightarrow} \mathrm{TP}_{j}\left(S \mid K, \mathbb{Z}_{p}\right)
$$

is an isomorphism.

Proof. It follows from Lemma 5.1 and the Tate orbit lemma [31, Lemma I.2.1] that

$$
\mathrm{TC}_{j}^{-}\left(S \mid K, \mathbb{Z}_{p}\right) \stackrel{\varphi}{\longrightarrow} \mathrm{TP}_{j}\left(S \mid K, \mathbb{Z}_{p}\right)
$$

is an isomorphism, for $j \geqslant 0$. We evaluate the common group for $j=2 k>0$, and consider the cases, where $S$ is of equal characteristic and of mixed characteristic, separately.

In the mixed characteristic case, the groups $\mathrm{THH}_{j}\left(S \mid K, \mathbb{Z}_{p}\right)$ for $j=2 k>0$ are zero by [20, Remark 2.4.2]. Hence, the homotopy fixed points spectral sequence

$$
E_{i, j}^{2}=H^{-i}\left(B \mathbb{T}, \mathrm{THH}_{j}\left(S \mid K, \mathbb{Z}_{p}\right)\right) \Rightarrow \mathrm{TC}_{i+j}^{-}\left(S \mid K, \mathbb{Z}_{p}\right)
$$


shows that the same is true for the groups $\mathrm{TC}_{j}^{-}\left(S \mid K, \mathbb{Z}_{p}\right)$.

If $S=k_{S}[\llbracket \pi]$ is of equal characteristic, then $\mathrm{TP}_{*}(S \mid K)$ is a graded algebra over the graded ring $\operatorname{TP}_{*}\left(\mathbb{F}_{p}\right)=\mathbb{Z}_{p}\left[v^{ \pm 1}\right]$, where $\operatorname{deg}(v)=-2$, and hence, is 2-periodic. We have canonical isomorphisms

$$
\mathrm{TP}_{0}(S \mid K) \stackrel{\text { can }}{\longleftarrow} \mathrm{TC}_{0}^{-}(S \mid K) \longleftarrow \mathrm{TF}_{0}(S \mid K ; p) \longrightarrow W\left(k_{S}\right),
$$

where the latter follows from Lemma 5.3 Under this identification, the two maps $\varphi$, can: $\mathrm{TC}_{0}^{-}(S \mid K) \rightarrow \mathrm{TP}_{0}(S \mid K)$ are given by the automorphisms of $W\left(k_{s}\right)$ induced by $\varphi$,id: $k_{S} \rightarrow k_{S}$, respectively. Finally, the maps $\varphi$, can: $\mathrm{TC}_{2}^{-}\left(\mathbb{F}_{p}\right) \rightarrow \mathrm{TP}_{2}\left(\mathbb{F}_{p}\right)$ are given by $\varphi\left(v^{-1}\right)=v^{-1}$ and $\operatorname{can}\left(v^{-1}\right)=p v^{-1}$; see [31, Section IV.4]. Hence, we find that for $j=2 k \geqslant 0$, the maps

$$
\mathrm{TC}_{j}^{-}(S \mid K) \stackrel{\varphi, \text { can }}{\longrightarrow} \mathrm{TP}_{j}(S \mid K)
$$

are respectively an isomorphism and $p^{k}$ times an isomorphism, so for $j=2 k>0$, their difference is an isomorphism, as stated.

Proof of Theorem B (2). We consider the diagram of spectra

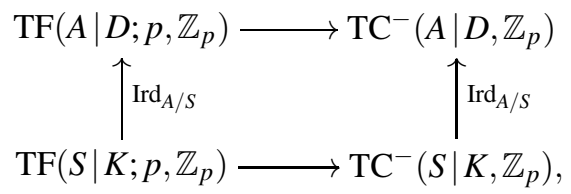

where the vertical maps are induced by the equivalence of cyclotomic spectra from part (1) of the theorem, and where the horizontal maps are the maps defined in the proof of Corollary 5.2 The diagram commutes by naturality of the horizontal maps, the vertical maps are equivalences, and, by Corollary 5.2 the horizontal maps induce equivalences of connective covers. The class $y=\operatorname{Ird}_{A / S}(1) \in \operatorname{TC}_{0}^{-}\left(A \mid D, \mathbb{Z}_{p}\right)$ defines a component in the mapping space

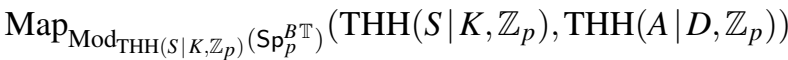

$$
\begin{aligned}
& \simeq \operatorname{Map}_{\mathrm{Sp}_{p}^{B \mathbb{T}}}\left(\mathbb{S}_{p}, \operatorname{THH}\left(A \mid D, \mathbb{Z}_{p}\right)\right) \simeq \Omega^{\infty} \operatorname{TC}^{-}\left(A \mid D, \mathbb{Z}_{p}\right)
\end{aligned}
$$

and any point in this component is an equivalence. Now, let $I_{A / S}$ be the map induced by extension of scalars along the inclusion of $S$ in $A$. It follows from Remark 1.9 and from the construction of the class $y$ that $I_{A / S}(1)=d \cdot y$. This shows that

$$
I_{A / S} \simeq d \cdot \operatorname{Ird}_{A / S}
$$

as maps of $\operatorname{THH}\left(S \mid K, \mathbb{Z}_{p}\right)$-modules in $\mathrm{Sp}_{p}^{B \mathbb{T}}$. We claim that this implies that also

$$
\operatorname{Tr}_{A / S} \simeq d \cdot \operatorname{Trd}_{A / S}
$$

as maps of $\operatorname{THH}\left(S \mid K, \mathbb{Z}_{p}\right)$-modules in $\mathrm{Sp}_{p}^{B \mathbb{T}}$. Indeed,

$$
d \cdot\left(\operatorname{Tr}_{A / S} \circ \operatorname{Ird}_{A / S}\right) \simeq \operatorname{Tr}_{A / S} \circ\left(d \cdot \operatorname{Ird}_{A / S}\right) \simeq \operatorname{Tr}_{A / S} \circ I_{A / S} \simeq d^{2} \cdot \mathrm{id},
$$


which implies that

$$
\operatorname{Tr}_{A / S} \circ \operatorname{Ird}_{A / S} \simeq d \cdot \mathrm{id},
$$

since $\mathrm{TC}_{0}^{-}\left(S \mid K, \mathbb{Z}_{p}\right)=W\left(k_{S}\right)$ is an integral domain. But we also have

$$
\left(d \cdot \operatorname{Trd}_{A / S}\right) \circ \operatorname{Ird}_{A / S} \simeq d \cdot\left(\operatorname{Trd}_{A / S} \circ \operatorname{Ird}_{A / S}\right) \simeq d \cdot \mathrm{id},
$$

and since $\operatorname{Ird}_{A / S}$ is an equivalence, the claim follows. This completes the proof.

Remark 5.5. The component in the mapping space

$$
\operatorname{Map}_{\operatorname{Mod}_{\operatorname{THH}\left(S \mid K, \mathbb{Z}_{p}\right)}\left(\operatorname{Sp}_{p}^{B \mathbb{T}}\right)}\left(\operatorname{THH}\left(A \mid D, \mathbb{Z}_{p}\right), \operatorname{THH}\left(S \mid K, \mathbb{Z}_{p}\right)\right)
$$

that contains the equivalence $\operatorname{Trd}_{A} / S$ is uniquely determined by the property that

$$
d \cdot \operatorname{Trd}_{A / S} \simeq \operatorname{Tr}_{A / S} .
$$

Indeed, the mapping space in question is simultaneously a torsor for the $\mathbb{E}_{\infty}$-monoids of endomorphisms of the domain and target, and their rings of components are both integral domains, being isomorphic to $W\left(k_{S}\right)$.

Proof of Theorem $A$ For all integers $j$, the equivalence of cyclotomic spectra $\operatorname{Trd}_{A / S}$ in Theorem B (1) induces an isomorphism

$$
\mathrm{TC}_{j}\left(A \mid D, \mathbb{Z}_{p}\right) \stackrel{\operatorname{Trd}_{A / S}}{\longrightarrow} \mathrm{TC}_{j}\left(S \mid K, \mathbb{Z}_{p}\right),
$$

and for $j \geqslant 1$, this induces the desired isomorphism

$$
K_{j}\left(D, \mathbb{Z}_{p}\right) \stackrel{\operatorname{Nrd}_{A / S}}{\longrightarrow} K_{j}\left(K, \mathbb{Z}_{p}\right)
$$

by Theorem 2.5 We claim that for $j \geqslant 1$, the former isomorphism is canonical and satisfies $d \cdot \operatorname{Trd}_{A / S}=\operatorname{Tr}_{A / S}$. Indeed, by Theorem 5.4 we have exact sequences

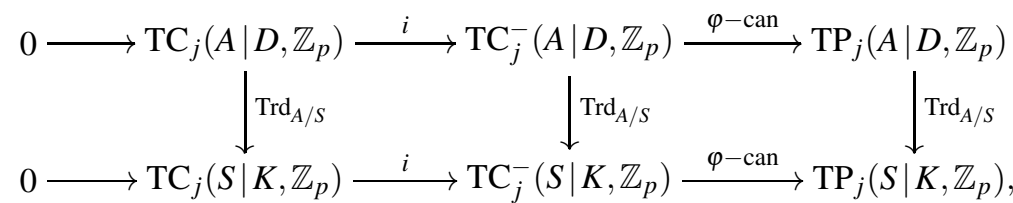

for $j \geqslant 1$ and odd, and by Theorem $\mathrm{B}$ (2), the middle and right-hand vertical maps are canonical and satisfy the desired identity. Theorem 5.4 shows similarly that for $j \geqslant 2$ and even, there are exact sequences

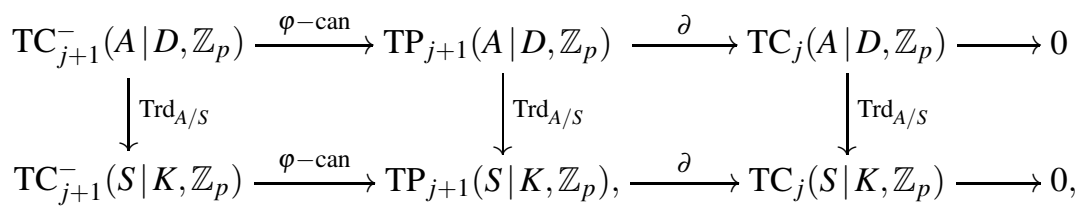

and by Theorem $B(2)$, the left-hand and middle vertical maps are canonical and satisfy the desired identity. This completes the proof. 
Lemma 5.6. The map $i_{*}: \mathrm{TC}_{j}\left(k_{S}, \mathbb{Z}_{p}\right) \rightarrow \mathrm{TC}_{j}\left(S, \mathbb{Z}_{p}\right)$ is zero for all integers $j$.

Proof. First, for $j=0$, we consider the following diagram with exact rows.

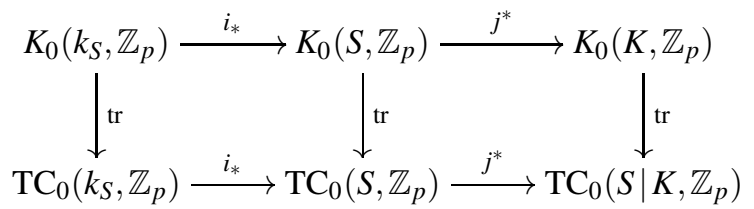

In the top row, the map $j^{*}$ is an isomorphism, since $S$ and $K$ both are local rings. Therefore, the map $i_{*}$ in the top row is zero, and since the left-hand and middle vertical maps are isomorphisms by [19. Theorem $\mathrm{D}]$, the map $i_{*}$ in the bottom row is zero, as stated. Next, we claim that the map

$$
\mathrm{TC}_{j}\left(S, \mathbb{Z}_{p}\right) \stackrel{i^{*}}{\longrightarrow} \mathrm{TC}_{j}\left(k_{S}, \mathbb{Z}_{p}\right)
$$

is surjective. Granting this, the lemma follows. Indeed, if $x=i^{*}(y)$, then

$$
i_{*}(x)=i_{*}(x \cdot 1)=i_{*}\left(i^{*}(y) \cdot 1\right)=y \cdot i_{*}(1),
$$

by the projection formula, and we have already proved that $i_{*}(1)=0$. The claims needs proof only if $j=0$ or $j=-1$, since the target of the map in question is zero, otherwise, and in these cases, the domain and target both are free $\mathbb{Z}_{p}$-modules of rank 1 . For $j=0$, the map is a $\mathbb{Z}_{p}$-algebra homomorphism, and therefore, it is necessarily an isomorphism. Finally, for $j=-1$, we consider the diagram

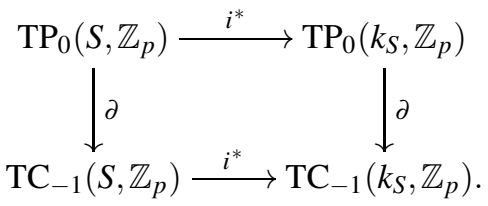

The top horizontal map is canonically identified with the map in the statement of Lemma 5.3, and hence, it is surjective. The right-hand vertical map also is surjective, since $\mathrm{TC}_{*}^{-}\left(k_{S}, \mathbb{Z}_{p}\right)$ is concentrated in even degrees by Bökstedt periodicity. It follows that the lower horizontal map is surjective, as claimed.

Corollary 5.7. The $\mathbb{Z}_{p}$-module $\mathrm{TC}_{0}\left(S \mid K, \mathbb{Z}_{p}\right)$ is free of rank 2.

Proof. By Lemma5.6, we have a short exact sequence

$$
0 \longrightarrow \mathrm{TC}_{0}\left(S, \mathbb{Z}_{p}\right) \stackrel{j^{*}}{\longrightarrow} \mathrm{TC}_{0}\left(S \mid K, \mathbb{Z}_{p}\right) \stackrel{\delta}{\longrightarrow} \mathrm{TC}_{-1}\left(k_{S}, \mathbb{Z}_{p}\right) \longrightarrow 0
$$

and, we have as already remarked, the left-hand term and the right-hand term both are free $\mathbb{Z}_{p}$-modules of rank 1 . 
Proof of Theorem $B(3)$. We consider the diagram

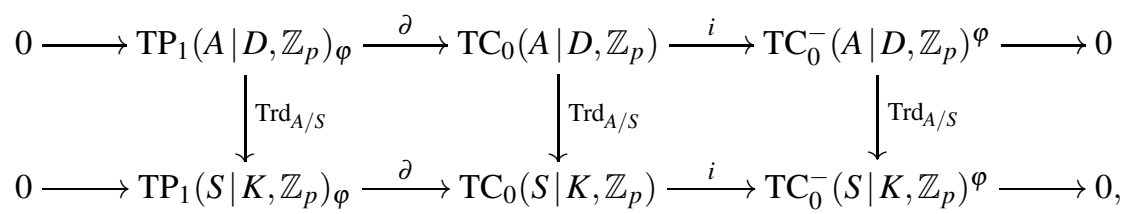

where $(-)^{\varphi}$ and $(-)_{\varphi}$ indicate the kernel and cokernel of $\varphi-$ can, respectively. The vertical maps are induced by the equivalence of cyclotomic spectra from part (1) of the theorem. Accordingly, the vertical maps are all isomorphisms and the diagram commutes. By Corollary 5.7, the middle terms are free $\mathbb{Z}_{p}$-modules of rank 2 , and we claim that the right-hand terms are free $\mathbb{Z}_{p}$-modules of rank 1 . Indeed, we have already identified $\mathrm{TC}_{0}^{-}\left(S \mid K, \mathbb{Z}_{p}\right)$ and $\mathrm{TP}_{0}\left(S \mid K, \mathbb{Z}_{p}\right)$ with $W\left(k_{S}\right)$ and the maps $\varphi$ and can with the maps induced by $\varphi$,id: $k_{S} \rightarrow k_{S}$, and since

$$
W\left(\mathbb{F}_{p}\right) \longrightarrow W\left(k_{S}\right) \stackrel{\text { id }}{\stackrel{W(\varphi)}{\longrightarrow}} W\left(k_{S}\right)
$$

is an equalizer of rings, the claim follows. We conclude that the left-hand terms are free $\mathbb{Z}_{p}$-modules of rank 1 as well.

We choose a basis $\left(e_{1}, e_{2}\right)$ of $\mathrm{TC}_{0}\left(S \mid K, \mathbb{Z}_{p}\right)$ such that $e_{1}$ is in the image of $\partial$ and such that $i\left(e_{2}\right)=1$, and let $\left(e_{1}^{\prime}, e_{2}^{\prime}\right)$ be the basis $\left(\operatorname{Trd}_{A / S}^{-1}\left(e_{1}\right), \operatorname{Trd}_{A / S}^{-1}\left(e_{2}\right)\right)$ of $\mathrm{TC}_{0}\left(A \mid D, \mathbb{Z}_{p}\right)$. By part (2) of the theorem, the matrix that represents

$$
\mathrm{TC}_{0}\left(A \mid D, \mathbb{Z}_{p}\right) \stackrel{\operatorname{Tr}_{A / S}}{\longrightarrow} \mathrm{TC}_{0}\left(S \mid K, \mathbb{Z}_{p}\right)
$$

with respect to these bases is of the form

$$
\left(\begin{array}{ll}
d & a \\
0 & d
\end{array}\right) \in M_{2}\left(\mathbb{Z}_{p}\right)
$$

We proceed to show that if $p$ divides $d$, then $d$ does not divide $a$, which proves part (3) of the theorem. To this end, we consider the commutative diagram

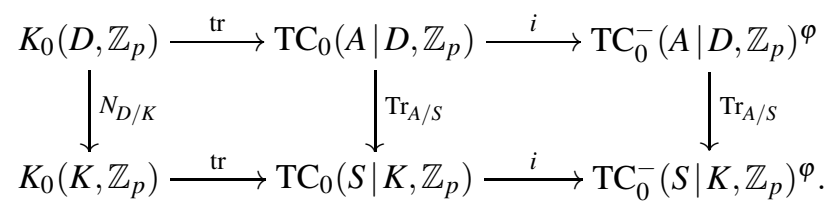

The left-hand terms are both free $\mathbb{Z}_{p}$-modules of rank 1 generated by the classes $[D]$ and $[K]$, and $N_{D / K}([D])=d^{2} \cdot[K]$. Moreover, the lower horizontal maps are ring homomorphisms, and hence, map the element $1=[K]$ of the lower left-hand term to the element 1 in the lower right-hand term. Since part (2) of the theorem shows that the right-hand vertical map is equal to $d \cdot \operatorname{Trd}_{A / S}$, we conclude that coordinates of the class $\operatorname{tr}([D]) \in \mathrm{TC}_{0}\left(A \mid D, \mathbb{Z}_{p}\right)$ with respect to the basis $\left(e_{1}^{\prime}, e_{2}^{\prime}\right)$ take the form

$$
\left(\begin{array}{l}
b \\
d
\end{array}\right) \in M_{2,1}\left(\mathbb{Z}_{p}\right)
$$


Hence, the coordinates of $\operatorname{Tr}_{A / S}(\operatorname{tr}([D]))$ with respect to the basis $\left(e_{1}, e_{2}\right)$ are

$$
\left(\begin{array}{ll}
d & a \\
0 & d
\end{array}\right)\left(\begin{array}{l}
b \\
d
\end{array}\right)=\left(\begin{array}{c}
d(a+b) \\
d^{2}
\end{array}\right) .
$$

It follows that $a+b \in \mathbb{Z}_{p}$ is divisible by $d$, since

$$
\operatorname{Tr}_{A / S}(\operatorname{tr}([D]))=\operatorname{tr}\left(N_{D / K}([D])=d^{2} \cdot \operatorname{tr}([K])=d^{2} \cdot 1\right.
$$

Therefore, $d$ divides $a$ if and only if $d$ divides $b$. We now consider the following commutative diagram with exact rows.

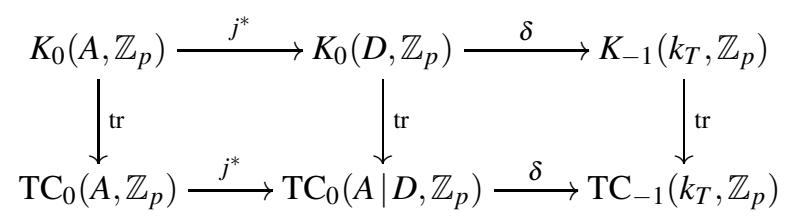

Since $A$ and $D$ are local rings, the map $j^{*}$ in the top row is an isomorphism between free $\mathbb{Z}_{p}$-modules of rank 1 . Moreover, the left-hand vertical map is an isomorphism by [19, Theorem D], since $A$ is a finite $\mathbb{Z}_{p}$-algebra. Hence, in the bottom row, the middle term is a free $\mathbb{Z}_{p}$-module of rank 2 , and the left-hand term and the right-hand term both are free $\mathbb{Z}_{p}$-modules of rank 1 . Therefore, in the bottom row, the map $j^{*}$ is injective, and its cokernel is a free $\mathbb{Z}_{p}$-module of rank 1 . We conclude that

$$
\operatorname{tr}([D])=\operatorname{tr}\left(j^{*}([A])\right)=j^{*}(\operatorname{tr}([A])) \in \mathrm{TC}_{0}\left(A \mid D, \mathbb{Z}_{p}\right)
$$

is not divisible by $p$. So if $p$ divides $d$, then $d$ does not divide $b$, and hence, $d$ does not divide $a$, as we wished to prove.

Corollary 5.8. If $p$ divides $d$, then the $G$-action on $\operatorname{THH}(A \mid D)$ is non-trivial.

Proof. If the $G$-action on $\operatorname{THH}(A \mid D)$ were trivial, then the diagram

$$
\begin{gathered}
\operatorname{THH}(A \mid D) \stackrel{f^{*}}{\longrightarrow} \operatorname{THH}\left(A \otimes_{S} T \mid D \otimes_{K} L\right)^{h G} \\
\operatorname{Trd}_{A \otimes_{S} T / T} \downarrow \uparrow \operatorname{Ird}_{A \otimes_{S} T / T} \\
\operatorname{THH}(S \mid K) \stackrel{f^{*}}{\longrightarrow} \operatorname{THH}(T \mid L)^{h G}
\end{gathered}
$$

would be meaningful and would define an equivalence

$$
\operatorname{THH}(A \mid D) \stackrel{\operatorname{Trd}_{A / S}}{\longrightarrow} \mathrm{THH}(S \mid K)
$$

of $\operatorname{THH}(S \mid K)$-modules in cyclotomic spectra such that $d \cdot \operatorname{Trd}_{A / S} \simeq \operatorname{Tr}_{A / S}$. However, if $p$ divides $d$, then Theorem B (3) shows that such an equivalence does not exist, so the $G$-action on $\operatorname{THH}(A \mid D)$ is necessarily non-trivial. 
Remark 5.9. The proof of Theorem 4.3 gives maps of exact sequences

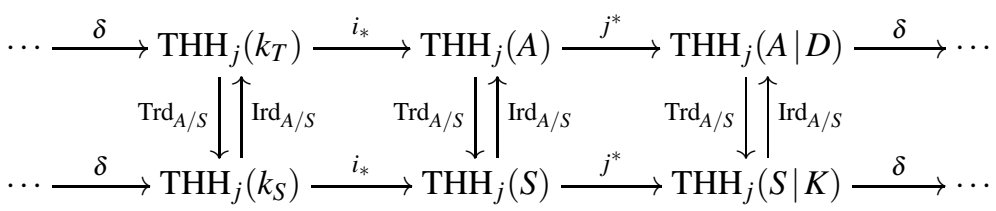

such that all maps are $\mathrm{THH}_{*}(S)$-linear and such that the right-hand vertical maps are isomorphisms defined in this paper. The analogous statement holds for the groups $\mathrm{TR}_{j}^{n}, \mathrm{TR}_{j}, \mathrm{TF}_{j}$, and for the respective groups with $p$-adic coefficients. We further expect that the left-hand vertical maps in the diagram agree with the maps $\operatorname{Tr}_{k_{T} / k_{S}}$ and $I_{k_{T} / k_{S}}$, respectively. Indeed, this would explain the appearance of the kernel of $\operatorname{Tr}_{k_{T} / k_{S}}$ in the calculations of $\mathrm{HH}_{*}\left(A / \mathbb{Z}_{p}\right)$ by the second author in [23, Theorem 3.5] and of $\operatorname{THH}_{*}\left(A, \mathbb{Z}_{p}\right)$ by the third author and Chan in [9, Theorem 5.1].

\section{References}

1. M. Artin, A. Grothendieck, and J. L. Verdier, Théorie des topos et cohomologie étale des schémas. tome 1: Théorie des topos, Séminaire de Géometrie Algébrique du Bois-Marie 1963-1964 (SGA 4), Lecture Notes in Math., vol. 269, Springer-Verlag, New York, 1972.

2. H. Bass, Algebraic K-theory, W. A. Benjamin, Inc., New York-Amsterdam, 1968.

3. M. Bökstedt, W.-C. Hsiang, and I. Madsen, The cyclotomic trace and algebraic K-theory of spaces, Invent. Math. 111 (1993), 465-540.

4. J. Borger, The basic geometry of Witt vectors, I: The affine case, Algebra Number Theory 5 (2011), 231-285.

5. The basic geometry of Witt vectors: II: Spaces, Math. Ann. 351 (2011), 877-933.

6. N. Bourbaki, Algebra I. Chapters 1-3. Translated from the French. Reprint of the 1989 English translation., Elements of Mathematics, Springer-Verlag, Berlin, 1998.

7. A. K. Bousfield, The localization of spectra with respect to homology, Topology 18 (1979), 257-281.

8. H. Cartan and S. Eilenberg, Homological algebra, Princeton University Press, 1956.

9. H. Y.-W. Chan and A. Lindenstrauss, Topological Hochschild homology of maximal orders in central simple $\mathbb{Q}$-algebras, Algebr. Geom. Topol. 19 (2019), 31-75.

10. V. Drinfeld, On the notion of geometric realization, Mosc. Math. J. 4 (2004), 619-626.

11. B. I. Dundas, K-theory theorems in topological cyclic homology, J. Pure Appl. Alg. 129 (1998), 23 33 .

12. B. I. Dundas and R. McCarthy, Topological Hochschild homology of ring functors and exact categories, J. Pure Appl. Alg. 109 (1996), 231-294.

13. T. Geisser and L. Hesselholt, Topological cyclic homology of schemes, Algebraic $K$-theory (Seattle, WA, 1997), Proc. Symp. Pure Math., vol. 67, Amer. Math. Soc., Providence, R.I., 1999, pp. 41-87.

14. On the K-theory of complete regular local $\mathbb{F}_{p}$-algebras, Topology 45 (2006), 475-493.

15. S. Geller and C. A. Weibel, Étale descent for Hochschild and cyclic homology, Comment. Math. Helv. 66 (1991), 368-388.

16. J. Giraud, Cohomologie non abélienne, Die Grundlehren der mathematischen Wissenschaften, vol. 179, Springer-Verlag, Berlin-New York, 1971.

17. L. Hesselholt, On the p-typical curves in Quillen's K-theory, Acta Math. 177 (1997), 1-53.

18. The big de Rham-Witt complex, Acta Math. 214 (2015), 135-207.

19. L. Hesselholt and I. Madsen, On the K-theory of finite algebras over Witt vectors of perfect fields, Topology 36 (1997), 29-102.

20.

21. B. Keller, Chain complexes and stable categories, Manuscripta Math. 67 (1990), 379-417.

22. A. Krause, The Tate spectral sequence for THH of log rings, Arbeitsgemeinschaft: Topological Cyclic Homology, Oberwolfach Rep., vol. 15, 2018. 
23. M. Larsen, Filtrations, mixed complexes, and cyclic homology in mixed characteristic, K-Theory 9 (1995), 173-198.

24. M. Larsen and A. Lindenstrauss, Topological Hochschild homology and the condition of HochschildKostant-Rosenberg, Comm. Algebra 29 (2001), 1627-1638.

25. A. Lindenstrauss, A relative spectral sequence for topological Hochschild homology of spectra, J. Pure Appl. Algebra 148 (2000), 77-88.

26. A. Lindenstrauss and I. Madsen, Topological Hochschild homology of number rings, Trans. Amer. Math. Soc. 352 (2000), 2179-2204.

27. S. MacLane, Categories for the working mathematician. Second edition, Graduate Texts in Mathematics, vol. 5, Springer-Verlag, New York, 1998.

28. M. A. Mandell, J. P. May, B. Shipley, and S. Schwede, Model categories of diagram spectra, Proc. London Math. Soc. 82 (2001), 441-512.

29. A. Merkurjev, K-theory of simple algebras, $K$-theory and algebraic geometry: connections with quadratic forms and division algebras (Santa Barbara, CA, 1992), Proc. Sympos. Pure Math., vol. 58 , Amer. Math. Soc., Providence, RI, 1995, pp. 65-83.

30. T. Nikolaus, Topological cyclic homology and cyclic K-theory, Arbeitsgemeinschaft: Topological Cyclic Homology, Oberwolfach Rep., vol. 15, 2018.

31. T. Nikolaus and P. Scholze, On topological cyclic homology, Acta Math. 221 (2018), 203-409.

32. D. Popescu, General Néron desingularization, Nagoya Math. J. 100 (1985), 97-126.

33. D. Quillen, Higher algebraic $K$-theory I, Algebraic $K$-theory I: Higher $K$-theories (Battelle Memorial Inst., Seattle, Washington, 1972), Lecture Notes in Math., vol. 341, Springer-Verlag, New York, 1973 , pp. 85-147.

34. I. Reiner, Maximal orders, London Mathematical Society Monographs, vol. 5, Academic Press, London-New York, 1975.

35. A. A. Suslin and A. V. Yufryakov, K-theory of local division algebras, Soviet Math. Dokl. 33 (1986), 794-798.

36. R. W. Thomason and T. Trobaugh, Higher algebraic K-theory of schemes and of derived categories, Grothendieck Festschrift, Volume III, Progress in Mathematics, vol. 88, 1990, pp. 247-435.

37. F. Waldhausen, Algebraic K-theory of spaces, Algebraic and geometric topology (New Brunswick, N. J., 1983), Lecture Notes in Math., vol. 1126, Springer-Verlag, Berlin, 1985, pp. 318-419. 